\title{
Analysis of Sequence Variability and Transcriptional Profile of Cannabinoid synthase Genes in Cannabis sativa L. Chemotypes with a Focus on Cannabichromenic acid synthase
}

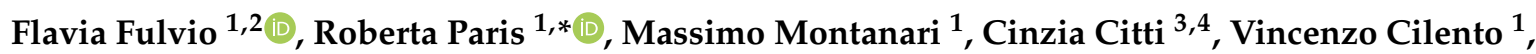 \\ Laura Bassolino ${ }^{1}{ }^{\mathbb{D}}$, Anna Moschella ${ }^{1}$, Ilaria Alberti ${ }^{5}$, Nicola Pecchioni ${ }^{6}$, Giuseppe Cannazza ${ }^{3,4}$ \\ and Giuseppe Mandolino ${ }^{1}$
}

Citation: Fulvio, F.; Paris, R.;

Montanari, M.; Citti, C.; Cilento, V.; Bassolino, L.; Moschella, A.; Alberti I.; Pecchioni, N.; Cannazza, G.; et al. Analysis of Sequence Variability and Transcriptional Profile of Cannabinoid synthase Genes in Cannabis sativa L. Chemotypes with a Focus on Cannabichromenic acid synthase. Plants 2021, 10, 1857. https://doi.org/ 10.3390/plants10091857

Academic Editor: Massimo Galbiati

Received: 4 August 2021

Accepted: 6 September 2021

Published: 8 September 2021

Publisher's Note: MDPI stays neutral with regard to jurisdictional claims in published maps and institutional affiliations.

Copyright: (C) 2021 by the authors Licensee MDPI, Basel, Switzerland. This article is an open access article distributed under the terms and conditions of the Creative Commons Attribution (CC BY) license (https:/ / creativecommons.org/licenses/by/ $4.0 /)$.
1 CREA-Research Centre for Cereal and Industrial Crops, Via di Corticella 133, 40128 Bologna, Italy; flavia.fulvio@crea.gov.it (F.F.); massimo.montanari@crea.gov.it (M.M.); vincenzo.cilento@edu.unito.it (V.C.); laura.bassolino@crea.gov.it (L.B.); anna.moschella@crea.gov.it (A.M.); giuseppe.mandolino@crea.gov.it (G.M.)

2 Department of Sciences of Agriculture, Food Natural Resources and Engineering, University of Foggia, Via Napoli 25, 71122 Foggia, Italy

3 CNR NANOTEC - Institute of Nanotechnology, Via Monteroni, 73100 Lecce, Italy; cinzia.citti@unimore.it (C.C.); giuseppe.cannazza@unimore.it (G.C.)

4 Department of Life Science, University of Modena and Reggio Emilia, Via G. Campi 103, 41125 Modena, Italy

5 CREA-Research Centre for Cereal and Industrial Crops, Via G. Amendola 82, 45100 Rovigo, Italy; ilaria.alberti@crea.gov.it

6 CREA-Research Centre for Cereal and Industrial Crops, S.S. 673 Km 25,200, 71122 Foggia, Italy; nicola.pecchioni@crea.gov.it

* Correspondence: roberta.paris@crea.gov.it

Abstract: Cannabis sativa L. has been long cultivated for its narcotic potential due to the accumulation of tetrahydrocannabinolic acid (THCA) in female inflorescences, but nowadays its production for fiber, seeds, edible oil and bioactive compounds has spread throughout the world. However, some hemp varieties still accumulate traces of residual THCA close to the $0.20 \%$ limit set by European Union, despite the functional gene encoding for THCA synthase (THCAS) is lacking. Even if some hypotheses have been produced, studies are often in disagreement especially on the role of the cannabichromenic acid synthase (CBCAS). In this work a set of European Cannabis genotypes, representative of all chemotypes, were investigated from a chemical and molecular point of view. Highly specific primer pairs were developed to allow an accurate distinction of different cannabinoid synthases genes. In addition to their use as markers to detect the presence of CBCAS at genomic level, they allowed the analysis of transcriptional profiles in hemp or marijuana plants. While the high level of transcription of THCAS and cannabidiolic acid synthase (CBDAS) clearly reflects the chemical phenotype of the plants, the low but stable transcriptional level of $C B C A S$ in all genotypes suggests that these genes are active and might contribute to the final amount of cannabinoids.

Keywords: cannabinoid synthase; SNPs; CBCAS; RT-qPCR

\section{Introduction}

Cannabis sativa L. is an annual, dioecious plant, characterized by the production of cannabinoids, terpenophenolic metabolites of great pharmaceutical interest, mainly synthetized and secreted in glandular trichomes of pistillate inflorescences.

Cannabinoids are produced by condensation of a phenolic moiety (usually olivetolic acid) with a terpenic one (geranylgeraniol-diphosphate). This reaction synthesizes cannabigerolic acid (CBGA), from which the other main cannabinoids are formed via enzymatic conversions.

Enzymatic cannabinoid biosynthesis is catalyzed by a number of oxidocyclases, among which the most prominent are the tetrahydrocannabinolic acid-, cannabidiolic acid- and 
cannabichromenic acid-synthase, leading to the accumulation of tetrahydrocannabinolic acid (THCA), cannabidiolic acid (CBDA) and cannabichromenic acid (CBCA) respectively [1]. These enzymes are thought to be poorly or completely non-functional in CBGAaccumulating plants [2].

Based on both the amount (expressed as\% weight/weight) of main cannabinoids and their ratio, the chemical phenotype (chemotype) of Cannabis plants can be classified from I to $\mathrm{V}$ [2].

THCA synthase (THCAS), $C B D A$ synthase (CBDAS) and $C B C A$ synthase (CBCAS) are members of the Berberine Bridge Enzyme (BBE)-like gene family, containing an $\mathrm{N}$-terminal signal peptide and a Flavin Adenin Dinucleotide (FAD) binding domain [3,4].

The genetics of cannabinoid synthesis has been studied for several years and different genes encoding these enzymes are known, each made of a single exon, with THCAS and CBCAS sharing $92 \%$ identity at amino acid level and $84 \%$ and $83 \%$ identity compared to CBDAS, respectively [3-7]. While THCAS and CBDAS have been widely studied at genetic and molecular level, little information is available on $C B C A S$ genes.

Originally, Kojoma et al. [8] obtained from hemp varieties gene sequences highly related to functional THCAS, putatively encoding for complete polypeptides, but differing for several single nucleotide polymorphisms (SNPs). They named these sequences fibertype THCAS genes, under the assumption that they were not functional and unable to synthesize cannabinoids [8]. Since then, a number of so-called fiber-type THCAS sequences have been found in both hemp and high THCA varieties [9,10].

In 2019, Laverty et al. [6] demonstrated that a fiber-type THCAS gene coded for a $71 \mathrm{kDa}$ CBCAS, capable of transforming the CBGA precursor into CBCA and that the accumulation of CBCA correlated with the transcriptional level of $C B C A S$ in various Cannabis tissues, with the highest level observed in female floral tissue.

More recently, a comprehensive clade-based classification of all cannabinoid oxidocyclases proposed the naming of all fiber-type THCAS as CBCAS [7] since they belonged to the same clade of the only functionally CBCAS characterized by Laverty et al. [6]. Based on these results today they are conventionally collectively referred to as $C B C A S$ and, according to this definition, in the present paper we will refer to all fiber-type THCAS sequences with a complete open reading frame as $C B C A S$.

Despite the name, further verification of their functionality in planta is needed and many authors claim for a meta-analysis to be conducted to verify that hypothesis $[7,11]$. Indeed, even though the CBCAS enzyme was biochemically characterized over 20 years ago [12] data on CBCAS are still scattered and somehow in disagreement. For example, one of the main inconsistencies with current evidence lies in previous analyses, where $\mathrm{CBCA}$ accumulation was demonstrated to be prominent during the juvenile stages of plant development, declining with maturity and irrespective of the chemotype at flowering [13].

Both the physiology of this enzyme and its relationship with the more studied CBDAS and THCAS are poorly understood and need clarification, especially in view of the possible contribution to the final chemotype.

Understanding whether $C B C A S$ sequences are present in each Cannabis variety or breeding material and the quantification of their transcription and potential translation in planta, would shed light on this important topic.

Several chemotype-associated PCR-based markers have been developed [10,14-16] to detect the presence of functional THCAS sequences, allowing to unambiguously distinguish between drug-type and fiber type materials. None of these markers amplify the $C B C A S$ sequences, despite their high similarity at nucleotide level and among them, the threeprimer marker system B1080/B1192 developed by Pacifico et al. [15], is fully associated with the chemotype, being able to discriminate chemotype I (THCA prevalent), II (both THCA and CBDA present in roughly similar amounts) and III (CBDA prevalent).

A number of complete and partially annotated genomic sequences have been published in the latest years [6,17-20] and information about the transcribed genes in different 
stages and tissues [21], as well as on putative regulators of secondary metabolites [22,23] is already known.

Despite the behavior as codominant alleles at a single locus observed in segregation analyses [24], THCAS and CBDAS genes have been recently mapped to separate loci in tight linkage on the same chromosome [6,19]. Moreover, in these loci there are multiple copies of THCAS- and CBDAS-related sequences. A few functional genes are expressed and therefore contribute to the final chemotype, while others are pseudogenes or partially functional sequences $[21,25,26]$.

Interestingly, many hemp inflorescences accumulate traces of residual THCA at concentrations close and sometimes above the limit of $0.20 \%$ of dry weight set by E.U., despite the lack of a functional THCAS gene in their genomes and years of breeding aimed at eliminating the accumulation of this cannabinoid. Some hypotheses have been proposed: the first one is that the CBDAS could produce with very low efficiency THCA from CBGA, due to its high similarity with THCAS [27]; alternatively, sequences corresponding to putative $C B C A S$ could be responsible for this residual production of THCA, small but apparently difficult to eliminate, since the substitutions at the protein level with functional THCAS are minimal and do not involve protein active sites or residues required for THCA synthesis [3,5]. Gaining a deeper knowledge of $C B C A S$ sequences and of their transcription levels could help to better define the process behind the synthesis of THCA in hemp genotypes as an unexpected by-product and consequently guide future breeding strategies.

In the present work, several fiber- and drug-type genotypes were investigated for the presence of $C B C A S$ sequences, assessing sequence variability and transcriptional levels. Markers were also developed to distinguish these sequences from the THCAS and CBDAS ones.

\section{Results}

\subsection{Genotyping}

Eleven hemp and two medical Cannabis genotypes (these last two currently used by pharmaceutical industry in Italy) were analyzed using the multiplex primer marker system B1080/B1192 [15].

A single amplification band at $1192 \mathrm{bp}$ was obtained for CINBOL (chemotype I, the only THCA-predominant variety), while for CINRO (chemotype II) both bands of expected size were amplified (Appendix A). For all hemp genotypes, the multiplex primer system amplified the 1080 band, with no distinction between chemotype III (Fibrante, Fibranova, Carmagnola, CS, Eletta Campana, Codimono, Carmaleonte and Futura 75), chemotype IV (Santhica 27 and Bernabeo) and Ermo (chemotype V), as already previously reported [15].

\subsection{Quantification of Major Cannabinoids}

The total amount of the main cannabinoids CBD, CBG and THC was measured by HPLC in female or monoecious hemp inflorescences harvested as reported in Table 1, about 4-5 weeks after full bloom, which would correspond, at least for day-length-sensitive genotypes, to the highest concentration of CBD or THC. Inflorescences of CINBOL and CINRO were collected earlier, to prevent an excessive accumulation of THC beyond the authorized limit for open field cultivation; therefore, total cannabinoid content was expected to be lower than at maturity (up to $18 \%$ in CINBOL and $15 \%$ in CINRO). 
Table 1. Quantitative results of total cannabinoid analysis expressed as the mean of 3 replicates. LOD (limit of detection): $0.001 \%$. LOQ (limit of quantification): $0.005 \%$. Information regarding inflorescences sampling period is expressed as days after sowing for hemp varieties, as days from onset flowering for CINBOL and CINRO. Results of genotyping obtained using the marker B1080/B1192 are given for each genotype. $B_{\mathrm{D}}$ and $B_{\mathrm{T}}$ refer to the allelic status as determined by the marker [25]. Data are expressed as percent of total inflorescence dry weight.

\begin{tabular}{|c|c|c|c|c|c|c|}
\hline Genotype & Sampling & CBD (\%) & CBG (\%) & THC (\%) & CBC (\%) & Marker Phenotype \\
\hline Santhica $27^{\mathrm{a}}$ & 123 & 0.26 & 1.55 & 0.04 & $<\mathrm{LOD}$ & $B_{D} / B_{D}$ \\
\hline Carmagnola & 151 & 5.32 & 0.28 & 0.16 & $<\mathrm{LOQ}$ & $B_{D} / B_{D}$ \\
\hline Bernabeo & 151 & 0.74 & 2.69 & 0.04 & $<\mathrm{LOQ}$ & $B_{D} / B_{D}$ \\
\hline Carmaleonte & 123 & 1.92 & 0.10 & 0.10 & $<\mathrm{LOQ}$ & $B_{D} / B_{D}$ \\
\hline $\mathrm{CS}$ & 151 & 5.49 & 0.20 & 0.17 & $<\mathrm{LOQ}$ & $B_{D} / B_{D}$ \\
\hline Ermo & 151 & 0.05 & $<$ LOD & $<\mathrm{LOD}$ & $<\mathrm{LOD}$ & $B_{D} / B_{D}$ \\
\hline Fibrante & 151 & 3.61 & 0.09 & 0.13 & $<\mathrm{LOQ}$ & $B_{D} / B_{D}$ \\
\hline Fibranova & 151 & 2.34 & 0.10 & 0.07 & $<\mathrm{LOQ}$ & $B_{D} / B_{D}$ \\
\hline Eletta Campana & 151 & 3.85 & 0.17 & 0.11 & $<\mathrm{LOQ}$ & $B_{D} / B_{D}$ \\
\hline Codimono & 123 & 4.02 & 0.15 & 0.14 & $<\mathrm{LOQ}$ & $B_{D} / B_{D}$ \\
\hline Futura $75^{a}$ & 123 & 2.34 & 0.12 & 0.07 & $<\mathrm{LOD}$ & $B_{D} / B_{D}$ \\
\hline CINBOL & 50 & 0.01 & 0.37 & 3.71 & 0.01 & $B_{T} / B_{T}$ \\
\hline CINRO & 50 & 2.43 & 0.27 & 1.57 & 0.02 & $B_{T} / B_{D}$ \\
\hline
\end{tabular}

${ }^{a}$ French varieties.

The varieties with the highest cannabinoid content were CS and Carmagnola, with $5.49 \%$ and $5.32 \%$ CBD/d.w, respectively (Table 1). The other chemotype III genotypes (usually characterized by relatively high amounts of CBD) showed a CBD content ranging from $4.02 \%$ in Codimono to $1.92 \%$ in Carmaleonte. In Santhica 27, one of the lowest amounts of CBD $(0.26 \%)$ was detected, followed by Ermo, which showed the lowest CBD content of all studied genotypes, as expected from its zero cannabinoids chemotype $\mathrm{V}$. A high CBD content was found in CINRO, while CINBOL showed relatively very low amount of CBD in its female inflorescences.

Total CBG ranged between $0.10 \%$ in Carmaleonte to $2.69 \% w / w$ in Bernabeo; it was not detected in Ermo.

All chemotype III genotypes had THC content below $0.20 \%$, the legal limit for industrial hemp in E.U., while, as expected, the two medical varieties CINBOL and CINRO exceeded the limit up to $3.71 \%$ and $1.57 \%$, respectively. Among hemp varieties, CS reached the highest residual THC content $(0.17 \%)$, followed by Carmagnola and Eletta Campana. The lowest THC content and the highest CBG content was registered for Santhica 27 and Bernabeo (chemotype IV), while no trace of THC was detected in Ermo. CBC was under the limit of quantification for all but the two medical varieties, with a higher content in the CINRO inflorescences $(0.02 \%)$.

\subsection{Diversity in $C B C A S$ Sequences}

Specific primers were designed following a search for the "fiber-type THCAS" reference sequence, AB212830 [8], in the genome assemblies available on NCBI in 2019, listed in Supplementary Table S1.

Complete Open Reading Frames (ORFs), pseudogenes and fragments of a few hundred base pairs were found, but only the complete sequences, with at least $99 \%$ query cover and more than $99 \%$ identity were further considered. At least one CBCAS homolog putatively encoding for a complete protein was retrieved from each genome assembly, except for Chemdog 91 and LA Confidential.

The alignment of the complete ORFs to the AB212830 sequence revealed the presence of three conserved SNPs, in position 13, 18 and 1628 from the starting codon. The same SNPs were always confirmed aligning the ORFs derived from genome assemblies to the other available putative $C B C A S$ genes deposited in public repositories. Based on this finding, a primer pair was designed (CBCAS-cds $\mathrm{Fw}$ and Rv, Table 2) including these three SNPs, which help to further differentiate these genes from THCAS and CBDAS. An alignment of 
one representative sequence for each genome against the reference sequence $\mathrm{AB} 212830$ is provided in Supplementary File S1, where these three polymorphisms are highlighted.

Table 2. List of primer pairs used for full length coding sequences (cds) amplification. Forward (Fw) and Reverse (Rv) primer sequences, amplicon length (bp) and references are given. Start and stop codons are underlined for each primer.

\begin{tabular}{|c|c|c|c|}
\hline Gene & Primer Sequence $\left(5^{\prime}-3^{\prime}\right)$ & Amplicon Length (bp) & Reference \\
\hline$C B C A S-c d s$ & $\begin{array}{l}\text { Fw: TGAAGAAAAATGAATTGCTCAACATTC } \\
\text { Rv: ACATAGTATGGGTAGATAATTAATGATGAC }\end{array}$ & 1666 & This work \\
\hline THCAS-cds & $\begin{array}{c}\text { Fw: ATGAATTGCTCAGCATTTTCCTT } \\
\text { Rv: ATGATGATGCGGTGGAAGA }{ }^{\mathrm{a}}\end{array}$ & 1635 & [25] \\
\hline$C B D A S-c d s$ & $\begin{array}{l}\text { Fw: ATGAAGTGCTCAACATTCTCCTT } \\
\text { Rv: TTAATGACGATGCCGTGGAA }\end{array}$ & 1635 & revised from [25] \\
\hline
\end{tabular}

${ }^{a}$ the stop codon is immediately after the end of the reverse primer.

In order to investigate the $C B C A S$ sequence variability within the set of 13 genotypes chosen in this work, PCR amplicons obtained with specific primers, covering the entire length of the coding sequence, were cloned and Sanger sequencing was performed on recombinant plasmids. All the putative $C B C A S$ sequences were obtained from a pool of genomic DNA from 10 individual plants for each genotype; sequencing led to the identification of both pseudogenes and full-length coding sequences. A total of $77 C B C A S$ sequences were obtained, among which 30 unique sequences with a new and complete ORF were submitted to GenBank with ID numbers: MW429515- MW429540, MW429551, MW561073- MW561075.

To properly classify these sequences, they were aligned to the sequences contained in Supplementary File S1 of the work by van Velzen and Schranz [7] and a phylogenetic analysis based on the nucleotide sequences showed that they belong to the clade A2, comprising the only functionally characterized $C B C A S$ and other putative $C B C A S$ (Figure 1).

One to six $C B C A S$ full cds were found in each Cannabis genotype analyzed in this work (Supplementary Table S2). The highest sequence variability was found in variety CS, with 6 different sequences, followed by Carmagnola, Carmaleonte and Fibrante, which have 5 different sequences each.

Several recurrent sequences were present in more than one genotype, such as MW429551 (Carmaleonte, Fibrante, Santhica 27, Bernabeo, Ermo, CINRO and CINBOL), MW429517 (CS, Futura 75, Carmaleonte), MW429518 (Carmagnola and Fibranova) and MW429528 (CS and Fibrante), whereas the other sequences were identified only in one out of 13 genotypes under investigation.

When compared with the AB212830 reference sequence, the alignment of the 30 new sequences revealed SNPs in 42 different positions of the cds, 28 of them resulting in either amino acid transitions or transversions. Sequences differed by a variable number of SNPs, from three (MW429517, MW429522, MW429528, MW429529, MW429534, MW429551) up to eight (MW429523).

The list of SNPs identified in the $C B C A S$ sequences with the corresponding position and any amino acid substitutions is reported in Supplementary Table S3.

The similarity of the new cds with the AB212830 ranges from 99.51\% (MW429520, MW429524) to $99.82 \%$ (MW429551), while the percentages of similarity with the THCAS (E33090) range from 95.72\% (MW429523) to 96.02\% (MW429534, MW429551). 


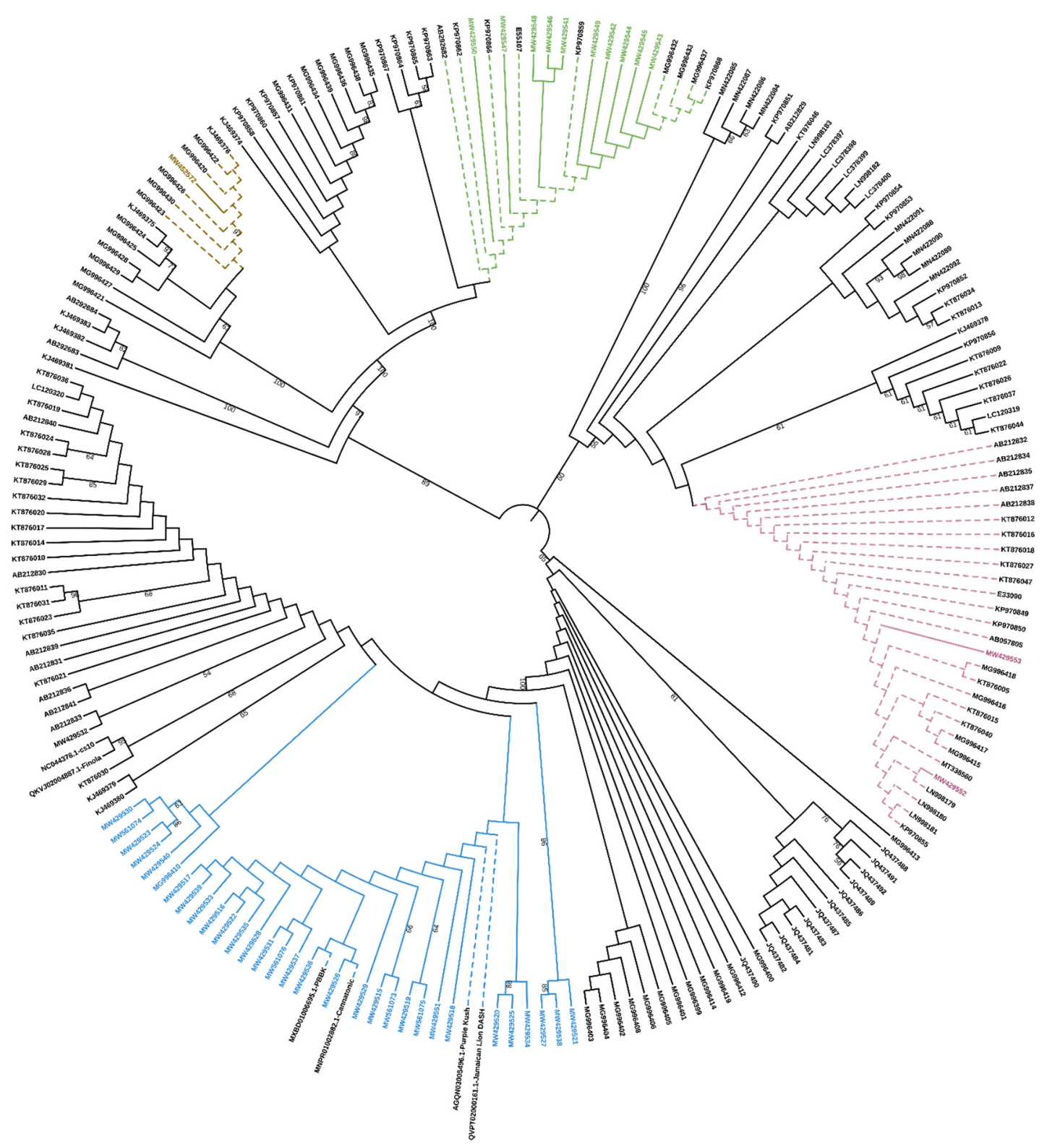

Figure 1. Phylogenetic tree analysis of 202 cannabinoid synthase genes from C. sativa at nucleotide level. The tree colors reflect the scheme presented by Van Velzen and Schranz in their Supplementary Figure S1. New CBCAS sequenced in the present work are in blue (clade A2), in pink new THCAS (clade A1) and in green new CBDAS sequences (clade, B, brown for clade B2-CBDAS). The optimal tree with the sum of branch length $=0.49420587$ is shown. Branches corresponding to partitions reproduced in less than $50 \%$ bootstrap replicates are collapsed. The percentage of replicate trees in which the associated taxa clustered together in the bootstrap test (1000 replicates) are shown next to the branches [28]. The evolutionary distances were computed using the Maximum Composite Likelihood method [29] and are in the units of the number of base substitutions per site. Codon positions included were 1st $+2 \mathrm{nd}+3 \mathrm{rd}+$ Noncoding. All ambiguous positions were removed for each sequence pair (pairwise deletion option). There were a total of 1906 positions in the final dataset.

\subsection{Diversity in THCAS Sequences}

Complete THCAS sequences were found only in the two medical Cannabis varieties, CINBOL and CINRO. All the eight sequences retrieved from CINRO (chemotype II) were identical to the reference functional THCAS sequence E33090. The variety CINBOL (chemotype I) showed higher variability, as three different sequences were found (E33090, MW429552, MW429553), out of seven sequenced complete cds. 
The possible presence of these sequences in databases was checked by BlastN against the standard NCBI non-redundant database, filtered by organism (Cannabis sativa, taxid: 3483) and no sequence with $100 \%$ identity was found.

The two novel sequences found in CINBOL variety are respectively $99.94 \%$ and $99.88 \%$ identical to E33090. Only one SNP $(\mathrm{A} \rightarrow 986 \mathrm{C})$ identified in MW429553 caused one amino acid change $(\mathrm{N} \rightarrow 328 \mathrm{~T})$ outside the three main domains of the putative translated protein.

\subsection{Diversity in CBDAS Sequences}

Among 65 sequenced clones, 10 unique sequences putatively encoding for functional proteins were identified. From one (Carmagnola, Futura 75, Santhica 27, Bernabeo, CINRO) up to four (Carmaleonte, Ermo) different complete coding sequences have been found in each genotype (Supplementary Table S2).

No complete CBDAS cds was identified in CINBOL (chemotype I), in agreement with other studies [9,30,31].

Compared to the reference CBDAS gene (GenBank accession E55107), from one to four SNPs were found in 11 different positions. These SNPs differentiating each sequence are listed in Supplementary Table S4 and seven of them cause amino acid substitutions. All sequences share a common SNP respect to the reference sequence in position 105, where $G$ was replaced by $T$, as already observed [25] This change is synonymous and therefore cannot affect the functionality of the resulting protein.

Overall, the percentages of similarity between all identified sequences and the reference CBDAS sequence (E55107) are very high.

The most divergent sequence from E55107 is the CBDAS isolated from the chemotype $\mathrm{V}$ accession Ermo (MW429543) (99.76\%), while the closest sequence is the MW429550 $(99.94 \%)$, detected in 10 out of 13 genotypes.

\subsection{Transcriptional Analysis of Cannabinoid synthase Genes}

The transcriptional level of the three classes of cannabinoid biosynthetic genes has been estimated by RT-qPCR in Cannabis inflorescences of all genotypes under study.

Given the high similarity of the target genes, the specificity of the designed primer pairs was accurately validated in three steps. Firstly, an in silico validation through blasting the primers to known reference sequences ensured that they perfectly matched exclusively sequences corresponding to the target genes. Only primers that generated perfect matches passed through the second validation step. This step was performed by PCR on different templates: genomic DNA, one plasmid containing the specific gene targeted by the primer pair (positive control) and one or more plasmids containing other non-target cannabinoid synthase genes (negative controls). Only the primer pairs that did not produce a band for the negative controls were brought to the next step (Appendix B). The same controls were then used in RT-qPCR analyses. In some cases, the specificity was gained after decreasing the primer concentration. Finally, amplification specificity was validated through melting curve analyses. Primer pairs showing a single high peak, indicating the absence of either nontarget amplifications or primer dimers, were selected for the RT-qPCR assay; the optimal settings ensuring only specific target amplification are reported in Table 3 . The melting curves for each specific amplicon are reported in Appendix B.

The transcriptional level of THCA synthase was analyzed in all 13 genotypes, but it could be detected and quantified only in CINBOL and CINRO varieties. The highest amount of THCA synthase transcripts was found in CINBOL (chemotype I, Figure 2a), where it was about 15 times higher than the transcription level detected in CINRO (chemotype II). On the contrary, $C B D A$ synthase gene was transcribed in almost all samples. The highest transcription level was found for CINRO (R.Q. = 4.9, Figure 2b), while it was undetectable in CINBOL, where no functional gene encoding for a $C B D A S$ was indeed found in this work. The CBDAS transcript levels varied among hemp varieties (R.Q. between 0.28 and 1.81), except for Santhica 27 where a very low transcript level was detected (R.Q. = 0.02). 
Table 3. List of primer pairs used for RT-qPCR. Forward (Fw) and reverse (Rv) primer sequences, amplicon length expressed in base pairs (bp), regression coefficient $\left(\mathrm{R}^{2}\right)$, PCR efficiency (Eff), annealing temperature (Ta) and usage concentrations $(\mu \mathrm{M})$ are given.

\begin{tabular}{|c|c|c|c|c|c|c|}
\hline Gene & Primer Sequence $\left(5^{\prime}-3^{\prime}\right)$ & $\begin{array}{l}\text { Amplicon } \\
\text { Length (bp) }\end{array}$ & $\mathbf{R}^{2}$ & Eff $(\%)$ & Ta & {$[\mu \mathrm{M}]$} \\
\hline CBDAS-RTqPCR & $\begin{array}{c}\text { Fw: GCAATACACACTTACTTCTCTTCAGTTTTC } \\
\text { Rv: ACGTAGTCTAACTTATCTTGAAAGCAC }\end{array}$ & 241 & 0.99 & 1.04 & 61.5 & 0.075 \\
\hline THCAS-RTqPCR & $\begin{array}{c}\text { Fw: AAAACTTCCTTAAATGCTTCTCAA } \\
\text { Rv: TAAAATAGTTGCTTGGATATGGGAGTT }\end{array}$ & 198 & 0.94 & 0.8 & 58 & 0.175 \\
\hline CBCAS-RTqPCR & $\begin{array}{c}\text { Fw: GCTCACGACTCACTTCAGAACTAG } \\
\text { Rv: GTAGAAGATGGTTGTATCAATCCAGCTC }\end{array}$ & 198 & 0.98 & 1.01 & 62 & 0.1 \\
\hline
\end{tabular}
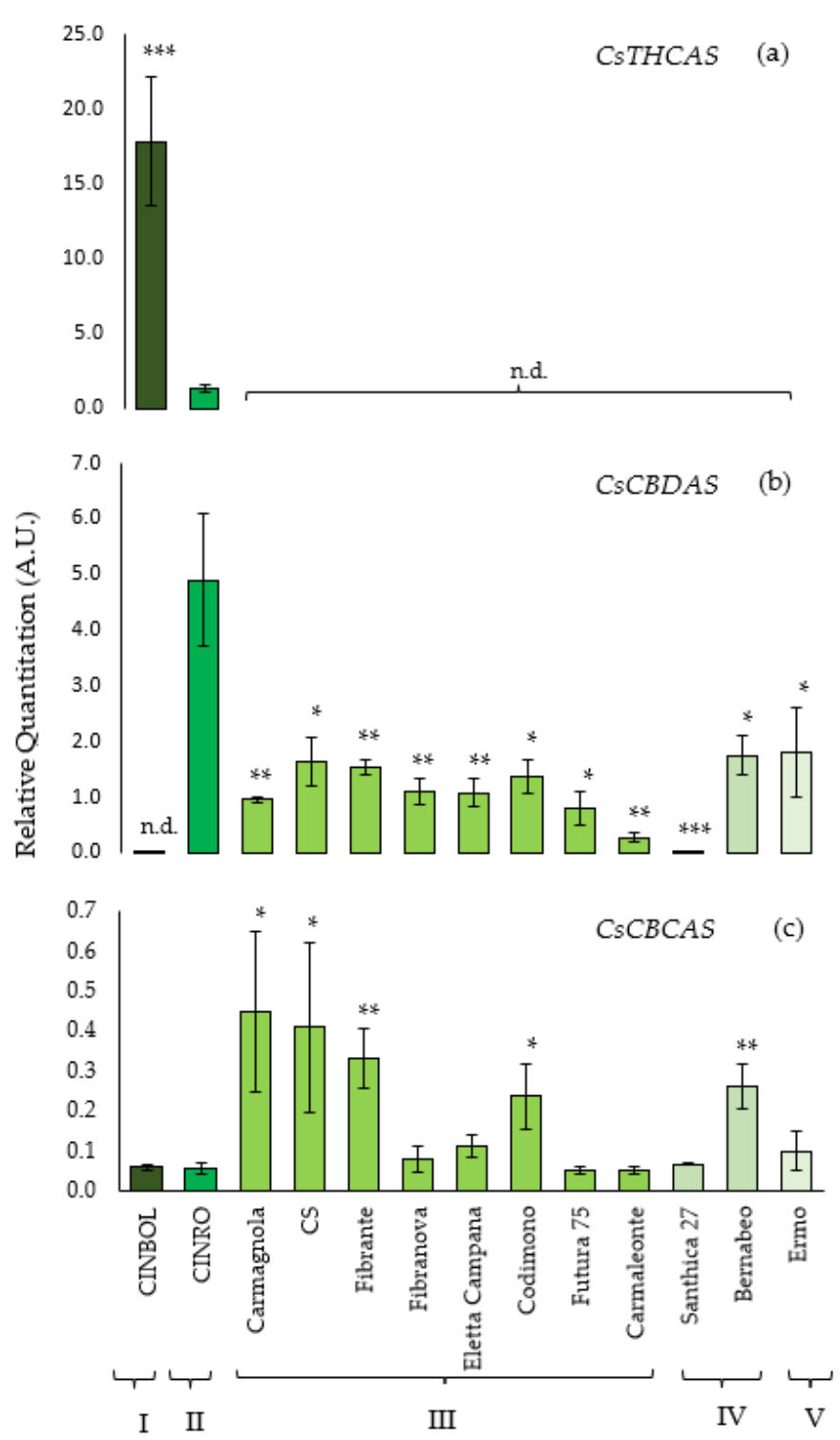

Figure 2. Relative Quantitation (R.Q.) of CsTHCAS (a), CsCBDAS (b), CsCBCAS (c) transcriptional levels in monoecious or female inflorescences. Y axis reports R.Q. expressed in Arbitrary Units (A.U.). Bars represent the standard errors of the mean of three biological replicates $(n=3)$. No detectable transcription levels are indicated as n.d. Below, for each genotype the corresponding chemotype is indicated (from I to V). Asterisks indicate Student's t-test statistically significant differences: ${ }^{*} p<0.05$; ** $p<0.01$ and ${ }^{* * *} p<0.001$. 
The transcription level of the putative $C B C A S$ genes has been investigated using the primers designed to avoid the amplification of non-target genes as THCAS and CBDAS (Table 3; Figure 2c) while amplifying all putative isoforms isolated in this work. In general, the $C B C A S$ sequences were transcribed in all genotypes under examination, with a lower transcription level compared to THCAS and CBDAS genes. Similar levels were observed for Carmagnola, CS, Fibrante, Codimono and Bernabeo; lower ones were detected in Ermo, Santhica 27, Carmaleonte, Futura 75, Eletta Campana, Fibranova, CINRO and CINBOL. Sequencing of RT-qPCR products further confirmed that the expressed genes of CINBOL and CINRO corresponded to the CBCAS genes (data not shown).

In CINRO, the only variety where all three genes are expressed, THCAS transcription levels (R.Q. = 1.32) are about four times lower than CBDAS levels (R.Q. = 4.90) and 24 times higher than CBCAS (R.Q. = 0.05). In CINBOL the expression of THCAS (R.Q. = 17.80) is about $300 \times$ the one of $C B C A S$ (R.Q. = 0.06).

In general, in industrial hemp genotypes, where THCAS could not be detected, CBDAS transcript levels were higher than $C B C A S$ ones (from $2 \times$ in Carmagnola up to $18 \times$ in Ermo). $C B C A S$ was transcribed more than $C B D A S$ only in Santhica 27 (Supplementary Table S5).

To the best of our knowledge, this is the first report about the transcription of putative $C B C A S$ genes related to the cannabinoid synthases and likely involved in CBCA synthesis and about a low but consistent presence of their mRNA in mature inflorescences in all tested samples, drug- but also hemp genotypes.

\section{Discussion}

The current knowledge of Cannabis sativa has been shaped by a growing number of studies regarding its therapeutic potential and the wide applications of its main bioactive compounds, mostly cannabinoids, turning this plant from a criminalized drug to a multibillion-dollar business in only a few years [32]. Recently the United Nations Commission on Narcotic Drugs agreed on the removal of Cannabis from Schedule I of the Controlled Substances Act and this is thought to boost research into medical Cannabis, giving the plant a well-deserved attention and recognizing the therapeutic properties of its bioactive metabolites.

As more studies on Eurasian landraces are required to unveil the genetic heterogeneity of the species [33], the present work focused on a set of Italian and French registered or patented Cannabis cultivars. The sequence variability and transcriptional levels of three cannabinoid synthases were investigated, focusing on $C B C A S$, reported for the first time in 2006 as fiber-type THCAS [8] and for many years neglected from the functional and genetic point of view.

In this work, many complete and putatively functional $C B C A S$ sequences were retrieved from both fiber- and drug-types genotypes, using a primer pair newly developed after in-silico analysis of different publicly available Cannabis assemblies.

Interestingly, a variable number of $C B C A S$ sequences was obtained from the genotypes under study, ranging from one in Codimono, CINRO and CINBOL to up to six different sequences in the case of CS, some of them recurring (e.g., MW429551, MW429517, MW429518, MW429528) in more than one variety, while others specific to one genotype suggesting a wide distribution and variability among genotypes.

Overall, these putative $C B C A S$ sequences confirmed a great conservation of the SNPs differentiating this class of genes from the THCAS genes, as previously reported [8-10]. This diversification from THCAS and the high degree of conservation among genotypes would support the hypothesis that these genes could exert a specific role in planta [6].

A role in the biosynthesis of CBCA was demonstrated only for one sequence [6]. Whether (and if) the expression of these sequences correlates with the ability of the different varieties to produce CBCA in some stage of their development and consequently if they should all be considered as actually involved in CBCA biosynthesis in planta, is still to be determined. This is true not only for the sequences identified in this work, but also for all other putative $C B C A S$ sequences released so far [7]. 
A recent phylogenetic analysis and subsequent classification of cannabinoid synthases has led to the definition of three different clades (A-C) [7]. The CBCAS retrieved in this work are included in the $\mathrm{A} 2$ clade, comprising full coding $C B C A S$ genes and sequences defined as "incomplete", "inactive" or "obscure" THCAS.

As expected, our $C B C A S$ are in a separate branch compared to other Sanger sequenced cds, due to the presence of three SNPs at positions 13,18 and $1628 \mathrm{bp}$, which deserve to be added to those previously identified as highly distinctive from THCAS $[8,10]$.

Here, an assay was specifically developed to identify and study the transcription of $C B C A S$, allowing to detect the low expression of these genes in all the analyzed genotypes.

Weakly expressed $C B C A S$ were found also in an RNA-seq experiment from female flower buds of nine Cannabis strains, where no detectable amounts of cannabichromene were found [34] In addition, Onofri et al., reported a small, yet detectable expression in pure-CBDA accessions [25].

According to Laverty et al. [6], the highest expression levels of $C B C A S$ were found in female flowers and trichomes rather than in leaves. However, the primers used in Laverty's work to analyze the CBCAS transcription by RT-qPCR are not specific and can actually target a $C B D A S$, as verified after BlastN vs. non redundant nucleotide database. On the other hand, de Meijer et al. [13] determined that CBCA accumulates predominantly in juvenile leaves and not in flowers. Taken together, these data made us sceptic about actuality and completeness of knowledge on $C B C A S$, that strongly encourages further analyses and verification of the function of these genes.

Grassa et al. [19] analyzed cannabinoid synthase genes expression by sequencing full length cDNA transcripts from high-CBD cultivars CBDRx (in leaves) and First Light (in flowers). No open reading frame for $C B C A S$ was found, probably due to the low transcriptional level of the gene in the analyzed samples, compared to other cannabinoid synthases.

Braich et al., using an RNA-seq approach, did not reveal CBCAS expression; however, this could be probably due to the misleading bioinformatics analyses of the short fragments deriving from paralogues and/or pseudogenes, all mapped to a same locus [21].

The analyses of transcriptional levels by using highly specific primers, as done in the present work, avoid the creation of misleading artifacts sometimes resulting by using short reads and coupled bioinformatics methods to analyze Cannabis transcriptomic massive outputs.

Different studies suggested the possibility that residual THCA in hemp plants is an apparently unavoidable by-product of CBDA synthesis by CBDAS; THCAS and CBDAS, in fact, have very similar kinetic characteristics $\left(\mathrm{V}_{\max }, \mathrm{K}_{\mathrm{M}}\right.$, turnover) and this might explain the simultaneous formation of these two cannabinoids from the common precursor CBGA $[27,28]$, though with different efficiencies. However, assuming this hypothesis was true, the issue about the existence of highly inbred lines accumulating practically only one single cannabinoid [24] would remain unexplained.

An alternative explanation is that, given their ubiquity in Cannabis germplasm, $C B C A S$ sequences likewise could be responsible for the low levels of THCA accumulation in hemp varieties at maturity, with occasional overcoming of the $0.20 \%$ d.w. THC threshold in some cultivations. The availability of specific markers, such as those presented in this work, could allow the counter-selection of these sequences during breeding, or their silencing by biotechnological strategies in a gene-specific and copy number-independent way, in order to definitively clarify the issue.

Comparing protein sequences of THCAS with CBCAS found in this work showed that there are no amino acid substitutions at residues necessary for the protein activity (from Arg110 to His114 and Cys176) or in residues whose substitution could affect its functionality, as demonstrated before [3,5]. Therefore, the possibility cannot be ruled out that the resulting CBCAS proteins, when translated, synthetize some THCA from CBGA precursor.

Based on our results, it appears that $C B C A S$ is transcribed at low levels in all genotypes and that the THCA amount is also low in hemp varieties; it cannot be overlooked that 
varieties accumulating higher residual THC (Carmagnola, CS, Fibrante, Codimono) also share higher amounts of $C B C A S$ transcripts (Table 1, Figure 2c).

Opposite to $C B C A S$, functional THCAS have been isolated in this work only from the medical varieties CINRO and CINBOL. In CINRO, only a THCAS sequence was retrieved from the genome, suggesting the presence of a single copy gene. Three different complete sequences of THCAS have been obtained from chemotype I CINBOL, suggesting the presence of at least two and up to three distinct copies of the gene. Both new sequences belong to the Subclade A1 from van Velzen and Schranz, with Type 1/1 (MW429552) and Type 1/2 (MW429553) differing by a single conservative substitution and therefore probably encoding for a functionally equivalent protein [25].

It has been suggested that reported variations in THCAS copy number had to be attributed to the use of promiscuous primers or to the unspecific inclusion of $C B C A S$ sequences instead of only functional THCAS [7]. In the present study, the selective amplification and sequencing of cds from clones and their phylogenetic analysis represent stronger evidence of the presence of more than one copy of THCAS gene per single genotype (CINBOL).

Moreover, a Copy Number Variation (CNV) between CINBOL and CINRO was supported by preliminary RT-qPCR data, showing that the amplification plot from the genomic DNA of CINBOL crossed the threshold line earlier than those from CINRO (data not shown), as expected if CINBOL had more copies of the target gene.

Whether or not this hypothetical CNV has a direct effect on the higher transcriptional level of THCAS and the higher amount of THCA in CINBOL in respect to CINRO is still to be determined. Other players like transcription factors or environmental conditions may be responsible for differences in transcriptional levels of these genes and cannabinoid accumulation [23].

Regarding the CBDA synthases class, a CBDAS gene has been sequenced (MW429549), with a SNP at position 1426, responsible for an amino acid substitution at position 476 of the enzyme (Pro $\rightarrow$ Ser). This substitution was already observed in Ermo and in another CBG-prevalent accession by Onofri et al. [25] and might result in a minimally functional CBDAS, unable to synthesize CBDA and leading to the accumulation of CBGA. This sequence probably accounts for chemotype IV when there are no other functional CBDAS, as in the case of Santhica 27 and Bernabeo.

\section{Materials and Methods}

\subsection{Plant Material and Samples Collection}

Nine Cannabis sativa hemp varieties, two hemp selections and two medical varieties were used in this work. Among hemp, six were dioecious (Carmagnola, CS, Fibrante, Fibranova, Eletta Campana, Bernabeo) and five monoecious (Ermo, Santhica 27, Carmaleonte, Codimono and Futura 75). Medical varieties CINBOL and CINRO (CPVO registration numbers 50407 and 50406, respectively) were developed at CREA, cultivated in authorized indoor facilities and clonally propagated by cuttings.

For DNA isolation, seeds of the eleven hemp genotypes were sown in a peat-type TRAYSUBSTRATE (Klasmann-Deilmann $\mathrm{Gmbh}$ ) and seedlings were grown in a plant growth chamber (Percival ${ }^{\circledR}$ AR-36LC8, Percival scientific, Inc. Perry, IA, USA), with a light/dark cycle of $18 / 6 \mathrm{~h}$, a temperature of $24{ }^{\circ} \mathrm{C}$ and $60 \%$ relative humidity. About 14 days after sowing, young leaves were collected from 10 different seedlings of the same genotype, pooled and conserved at $-80^{\circ} \mathrm{C}$ until DNA extraction.

For RNA isolation, the seeds of hemp genotypes were sown on the 19 April 2019 and cultivated in open field in CREA-CI experimental station of Rovigo (GPS coordinates: 45.078722/11.766035). Three biological replicates were collected from three different plants for each genotype, each consisting of three female or monoecious $10 \mathrm{~cm}$ apical flowers (comprising apical flower and floral leaves) sampled 123 days after sowing from the monoecious varieties and 151 days after sowing from the dioecious ones.

Samples (Figure 3) were immediately frozen in liquid nitrogen and stored at $-80^{\circ} \mathrm{C}$. 


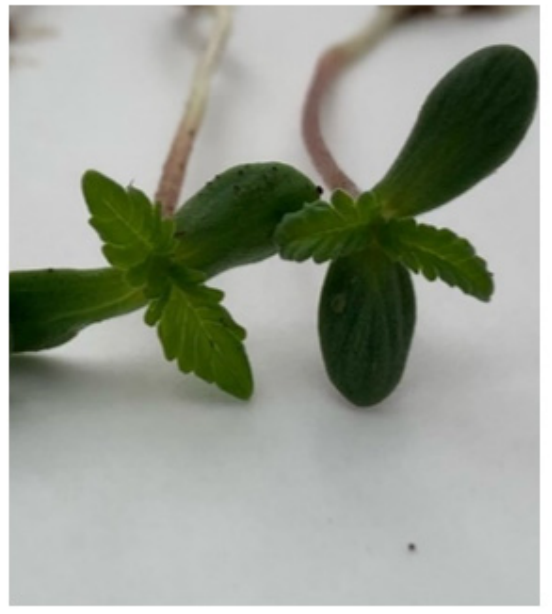

(a)

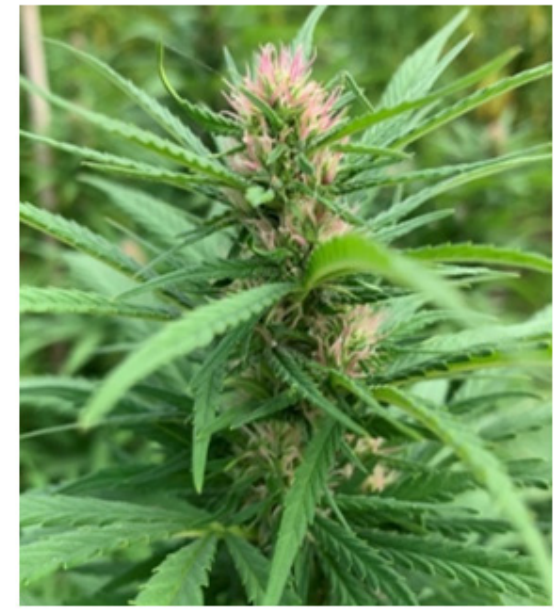

(b)

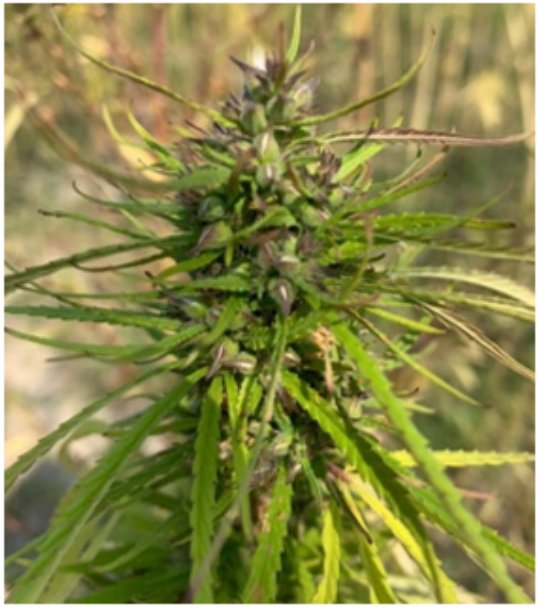

(c)

Figure 3. (a) Cannabis samples collected for DNA extraction, molecular cloning and sequencing. (b) CINRO female inflorescence 50 days after onset flowering. (c) Chemotype III dioecious genotype female inflorescence 151 days from sowing.

Medical varieties with high THCA CINRO and CINBOL were also grown outdoor starting from cuttings from mother plants. For DNA extraction expanded leaves were collected from young, rooted cuttings; for RNA extraction, inflorescences were collected 50 days after the start of flowering, in the attempt to prevent an accumulation of THCA beyond the limit authorized for open field cultivation in Rovigo station. Samples were conserved as described above.

For chemical analysis, the same inflorescences were used after lyophilization.

\subsection{LC-UV Analysis}

For quantitative analysis of standard cannabinoids, namely CBDA, CBGA, THCA, CBD, CBG, $\Delta^{9}$-THC, $\Delta^{8}$-THC and CBC, samples (500 mg inflorescence of each variety, finely powdered) were prepared and analyzed by liquid chromatography coupled with UV detection (HPLC-UV) according to the protocol of the German Pharmacopoeia as previously reported [35,36]. Briefly, the extraction in analytical grade ethanol 96\% (Carlo Erba, Milan, Italy) was carried out in three cycles with progressively decreasing volumes of solvent $(20,12.5$ and $12.5 \mathrm{~mL})$ under magnetic stirring for $15 \mathrm{~min}$ each. After collecting the liquid fraction in a volumetric flask, the extract was brought to $50 \mathrm{~mL}$ final volume with fresh ethanol. A $1 \mathrm{~mL}$ aliquot was filtered through a $0.45 \mu \mathrm{m}$ syringe filter, diluted 1:10 with acetonitrile and injected into the chromatographic apparatus $(5 \mu \mathrm{L})$. The chromatographic separation was performed on a Vanquish Core UHPLC system (Thermo Fisher Scientific, Waltham, MA, USA) equipped with a vacuum degasser, a binary pump, a thermostated autosampler at $4{ }^{\circ} \mathrm{C}$, a thermostated column compartment set at $30^{\circ} \mathrm{C}$ and a diode array detector. The analyses were carried out following the method reported in previous works [37-39] with slight modifications and acquired with the Chromeleon 7.3 Data System software (Thermo Fisher Scientific) following the UV signal registered at $228 \mathrm{~nm}$. The column employed was a Poroshell 120 C18 column (Poroshell 120 EC-C18, $3.0 \times 150 \mathrm{~mm}, 2.7 \mu \mathrm{m}$ ) (Agilent, Milan, Italy) and the mobile phase consisted of water (solvent A) and acetonitrile (solvent B) both with $0.1 \%$ formic acid. A linear gradient from 5 to $95 \%$ B was set over $20 \mathrm{~min}$, then the column was washed with $95 \%$ B for $5 \mathrm{~min}$ and reconstituted with the initial mobile phase $(5 \% \mathrm{~B})$ for other 5 min bringing the total run time to $30 \mathrm{~min}$.

Calibration curves were individually prepared in acetonitrile for each cannabinoid standard from $1 \mathrm{mg} / \mathrm{mL}$ stock solutions (Cerilliant, Sigma Aldrich, Milan, Italy) and linearity was assessed in the range $0.05-5.00 \mu \mathrm{g} / \mathrm{mL}$ for all cannabinoids and also in the range $5.0-50 \mu \mathrm{g} / \mathrm{mL}$ for CBD in the case of CBD-rich, chemotype III, genotypes $\left(R^{2}>0.999\right)$. The total cannabinoid content was calculated according to Baratta et al. [37]. Three injec- 
tions were performed for each sample and results are given as $\%(w / w)$, expressed as mean of replicates $(n=3)$. The limit of detection (LOD) was $0.001 \%$ and limit of quantification (LOQ) was $0.005 \%$.

\subsection{DNA Isolation and Genotyping PCR Analysis}

For DNA isolation, $100 \mathrm{mg}$ of frozen pooled leaves were finely ground by physical treatment with steel beads in a Tissue Lyser II (Qiagen) at $30 \mathrm{~Hz}$ for five minutes and extracted using the Invisorb ${ }^{\circledR}$ Spin Plant Mini Kit (STRATEC molecular Gmbh, Berlin, Germany) according to the manufacturer's instructions. DNA was eluted in $100 \mu \mathrm{L}$ sterile water, quantified at the Infinite $200 \mathrm{PRO}$ spectrophotometer (TECAN) and diluted to $10 \mathrm{ng} / \mu \mathrm{L}$.

The three-primers multiplex system used to genotype the $C$. sativa plants at the $B$ locus [15] is detailed in Appendix A and generated either a $1081 \mathrm{bp}$ (presence of a functional $C B D A S$ ), a $1192 \mathrm{bp}$ (presence of a functional THCAS) or both fragments (presence of both functional genes).

\subsection{RNA Isolation and cDNA Synthesis}

For RNA isolation, $100 \mathrm{mg}$ frozen samples were finely ground and extracted using the Spectrum Plant Total RNA Kit (Sigma Aldrich, Merck Life Science S.r.l., Milan, Italy). Total RNA was eluted in $50 \mu \mathrm{L}$ of DEPC-treated water and spectrophotometrically quantified. Five hundred ng of total RNA were treated with 1 unit of DNAse I Amplification Grade (Sigma-Aldrich, Merk Life Science S.r.l., Milan, Italy) and retrotranscribed with the High-Capacity RNA to cDNA kit (Thermo Fisher Scientific, Waltham, MA, USA) according to manufacturer's instructions.

\subsection{PCR Amplification, Cloning and Sequencing}

THCAS- and CBDAS-specific primers from Onofri et al. [25], with some modifications as specified in Table 2, were used in this work to isolate the complete coding sequences of the genes.

For a specific amplification of the $C B C A S$ genes, a search on Cannabis assemblies (available at: https://www.ncbi.nlm.nih.gov/assembly/?term=cannabis, accessed on 19 December 2019) was performed in order to identify conserved regions allowing the discrimination between active and "inactive" forms. Once identified, primers were designed using the software Primer3 [40,41].

The amplification reactions were performed on $20 \mathrm{ng}$ of template DNA using the Invitrogen $^{\mathrm{TM}}$ Platinum ${ }^{\mathrm{TM}}$ SuperFi ${ }^{\mathrm{TM}}$ polymerase (Thermo Fisher Scientific, Waltham, MA, USA), which has a reported fidelity $>300$ times higher than Taq, in $50 \mu \mathrm{L}$ reaction. PCR products, purified using the NucleoSpin ${ }^{\circledR} \mathrm{Gel}$ and PCR Clean-up kit (Macherey-Nagel GmbH \& Co. KG, D Düren, Germany), were cloned in pJET1.2 plasmid vectors (CloneJET PCR Cloning Kit, Thermo Fisher Scientific, Waltham, MA, USA) and transferred in Escherichia coli $\mathrm{DH} 5 \alpha$ cells in accordance with the manufacturer's protocol. Properly transformed cells were selected for ampicillin resistance $(100 \mu \mathrm{g} / \mathrm{mL}$ final concentration) on LB -agar medium and colonies were screened by PCR, using the protocol and primers suggested in the cloning kit.

For each genotype, up to eight plasmids for each gene were isolated using the PureLink®Quick Plasmid Miniprep Kit (Thermo Fisher Scientific, Waltham, MA, USA). The inserts were then sequenced according to the Sanger method (BMR Genomics Srl, Padova, Italy), using the pJET 1.2 sequencing primers (pJET 1.2 forward: CGACTCACTATAGGGAGAGCGGC; pJET 1.2 reverse: AAGAACATCGATTTTCCATGGCAG).

Only complete coding sequences were further considered in this work. The variability of THCA and CBDA synthases was evaluated firstly by aligning the sequences from each individual genotype in order to find unique single sequences. These were aligned with the two reference sequences of THCA- and CBDA-synthase (E33090 and E55107) and, finally, compared across the different genotypes. The sequence comparison at nucleotide 
and amino acid level was carried out using MUSCLE 3.8 (available at: https:/ /www. ebi.ac.uk/Tools/msa/muscle/ accessed on 8 March 2021). For phylogenetic analysis the evolutionary history was inferred using the Neighbor-Joining method in the MEGA-X software $[29,42]$. The final figure of NJ DIRs tree was obtained by using the iTOL tree editor (The iTOL Platform).

\subsection{Transcriptional Analysis of Cannabinoid synthases}

The transcriptional levels of the $C B D A-, T H C A$ and $C B C A S$ and of three candidate reference genes CsActin, CsRAN (Ras-related Nuclear protein) and CsClathrin [43] were measured by reverse transcription quantitative real time PCR (RT-qPCR) using a RotorGene 6000 (Corbett) and SYBR Green chemistry.

Each reaction contained $3 \mu \mathrm{L}$ of a 1:9 dilution of cDNA, $5 \mu \mathrm{L}$ of Power $\mathrm{Up}^{\circledR} \mathrm{SYBR}$ master mix (Thermo Fisher Scientific), highly specific primers and RNA-free water to a final volume of $10 \mu \mathrm{L}$. Primer pairs, optimized conditions and annealing temperatures are listed in Table 3. Primer design and the optimization steps performed to assure the high specificity of amplification were performed following Pagliarani et al. [44] and a detailed description is reported in Appendix B. Further details of RT-qPCR conditions are reported in Supplementary File S2 following the Minimum Information for publication of Quantitative Real-Time PCR Experiments (MIQE) guidelines [45,46].

Different amplification conditions were used depending on the annealing temperature of the primers used: when this was below $60^{\circ} \mathrm{C}$, a two-step method was used, consisting of $15 \mathrm{~s}$ at variable temperature for annealing and $1 \mathrm{~min}$ at $72{ }^{\circ} \mathrm{C}$ for extension; when this was above $60^{\circ} \mathrm{C}$, a one-step method was used, consisting of $60 \mathrm{~s}$ at the desired temperature in which both annealing and extension are performed. Finally, a heat dissociation protocol (from $60^{\circ} \mathrm{C}$ to $99^{\circ} \mathrm{C}$ ) was performed and a dissociation curve for each sample was generated. Three biological replicates were analyzed for each genotype, which in turn were tested in three technical replicates.

A standard curve was added in all assays, both for target and for reference genes. Standard curves were made of 5 points, prepared as four 1:4 serial dilutions of a 1:3 dilution of the cDNA (500 ng).

To verify the specificity of reaction, each assay included also several negative controls as specified in Appendix B.

The amplification efficiency (E) of each primer pair was estimated using the slope of the regression line, according to the equation: $\mathrm{E}=10^{\wedge}(-1 /$ slope $)-1$. The transcriptional level stability across samples of CsActin, CsRAN and CsClathrin was verified by software RefFinder [47] and, according to results, CsClathrin and CsRAN were selected as reference genes.

Raw data for target and reference genes were transformed using the 'Standard Curve Method'; the transcripts level of target genes was normalized to the geometric mean of the transcripts level of CsClathrin and CsRAN and reported as Relative Quantitation (RQ) of transcriptional levels [48] expressed in Arbitrary Units (A.U.). Finally, the standard error of the mean of three biological replicates was calculated and reported in the graphs as error bar. Comparison between genotypes vs CINRO for each gene transcript levels was done using Student's t-test with log-transformed expression data.

\section{Conclusions}

This work describes for the first time sequence variability of cannabinoid synthases coding sequences and their transcriptional profiles in a set of Cannabis genotypes, belonging to five different chemotypes. Highly specific primers were developed, to be used as robust markers for univocal identification and analysis of THCAS, CBDAS and CBCAS in different Cannabis tissues. While the higher levels of transcription detected for THCAS in respect to $C B D A S$ can be related to the different end use of THCA-rich varieties compared with fiber varieties, the meaning of the low but almost constant level of transcription of $C B C A S$ still remains to be fully understood. The existence of a cluster of $C B C A$ synthases separated 
from functional THCA synthases suggest a different role in the plant as evolution and/or selection has created a whole set of highly conserved sequences. According to our data, $C B C A S$ could play a role for producing residual THCA in CBD-predominant genotypes, but more research is still needed to confirm this hypothesis.

Altogether, the results reported here and the assay developed will pave the way for more accurate functional studies in planta of these gene families and of how their reciprocal relationships can influence the quantitative component of chemotype.

Supplementary Materials: The following are available online at https:/ /www.mdpi.com/article/ 10.3390/plants10091857/s1, Supplementary File S1: Alignment of CBCAS nucleotide sequences; Supplementary File S2: detailed MIQE checklist; Supplementary Tables S1-S4; Table S1: Summary of features of the Cannabis sativa genomes accessed, Table S2: Sequence variation within Cannabis germplasm, Table S3: SNPs found in the CBCAS sequences found in Cannabis germplasm, Table S4: SNPs found in the CBDAS sequences in Cannabis germplasm, Table S5: Ratio between CBDAS and $C B C A S$ relative quantitation.

Author Contributions: Conceptualization, F.F., R.P. and G.M.; methodology, F.F., R.P., G.M.; formal analysis, F.F., V.C., C.C., L.B.; investigation, F.F., R.P., C.C.; resources, M.M., I.A., A.M.; writingoriginal draft preparation, F.F., R.P., G.M.; writing - review and editing, all authors.; supervision, R.P., G.M.; project administration, R.P., G.M.; funding acquisition, R.P., G.M., N.P., G.C. All authors have read and agreed to the published version of the manuscript.

Funding: This research was funded by MAIDET research project "Metodi analitici innovativi per la determinazione del THC in matrici vegetali", funded by the Ministero delle Politiche Agricole Alimentari e Forestali (MIPAAF), grant decree D.M.34176/7303/2017 and UNIHEMP research project "Use of iNdustrIal Hemp biomass for Energy and new biocheMicals Production" (ARS01_00668) funded by European Regional Development Fund (within the PON R\&I 2017-2020-Axis 2-Action II-OS 1.b). Grant decree UNIHEMP prot. no. 2016 of 27/07/2018; CUP B76C18000520005.

Data Availability Statement: Data is contained within the article and Supplementary Materials.

Acknowledgments: F.F. and R.P. would like to thank Virna Benazzi for her help in collecting plant material, Gianmaria Magagnini for his contribution in field operation and his helpful support during sampling, as well as useful and never trivial conversations and Laura Righetti for reading and revised the manuscript and for kind advice.

Conflicts of Interest: The authors declare no conflict of interest.

\section{Appendix A}

Chemotype assessment.

Table A1. Primer sequences used for chemotype assessment.

\begin{tabular}{cc}
\hline Primer Name & Sequence $\left(5^{\prime}->\mathbf{3}^{\prime}\right)$ \\
\hline THCAS $/$ CBDAS fw & AAGAAAGTTGGCTTGCAG \\
CBDAS rev & ATCCAGTTTAGATGCTTTTCGT \\
THCAS rev & TTAGGACTCGCATGATTAGTTTTTC \\
\hline
\end{tabular}


Table A2. PCR reaction set up for three-primers marker system.

\begin{tabular}{cc}
\hline Component & Final Concentration \\
\hline Water & $\mathrm{Up}$ to $25 \mu \mathrm{L}$ \\
$10 \times$ PCR Buffer, $-\mathrm{Mg}$ & $1 \times$ \\
$50 \mathrm{mM}$ MgCl2 & $1.5 \mathrm{mM}$ \\
$10 \mathrm{mM}$ dNTP Mix & $0.2 \mathrm{mM}$ each \\
$10 \mu \mathrm{M}$ forward primer & $0.2 \mu \mathrm{M}$ \\
$10 \mu \mathrm{M}$ reverse primer 1 & $0.2 \mu \mathrm{M}$ \\
$10 \mu \mathrm{M}$ reverse primer 2 & $0.2 \mu \mathrm{M}$ \\
Template DNA & $10 \mathrm{ng}$ \\
Taq DNA Polymerase $(5 \mathrm{U} / \mu \mathrm{L})$ & $0.04 \mathrm{U} / \mu \mathrm{L}$ \\
\hline
\end{tabular}

Table A3. PCR program for three-primers marker system.

\begin{tabular}{cccc}
\hline Step & Temperature & Time & Cycles \\
\hline Initial denaturation & $94^{\circ} \mathrm{C}$ & $3 \mathrm{~min}$ & 1 \\
Denaturation & $94^{\circ} \mathrm{C}$ & $45 \mathrm{~s}$ & 32 \\
Annealing & $62^{\circ} \mathrm{C}$ & $1 \mathrm{~min}$ & \\
Extension & $72^{\circ} \mathrm{C}$ & $2 \mathrm{~min}$ & 1 \\
Final extension & $72^{\circ} \mathrm{C}$ & $10 \mathrm{~min}$ & \\
\hline
\end{tabular}

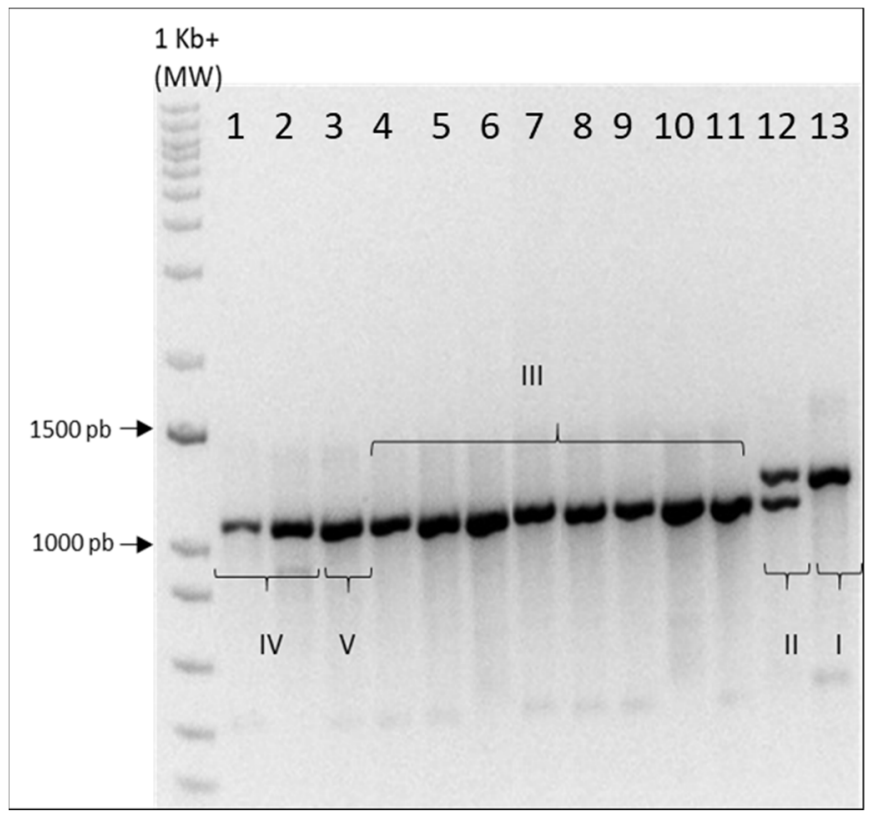

Figure A1. Chemotype determination. The respective chemotypes are indicated for each genotype (I-V). From left to right the genotypes are: 1, Santhica 27; 2, Bernabeo; 3, Ermo; 4, Fibranova; 5, Eletta Campana; 6, Futura 75; 7, Carmagnola; 8, Carmaleonte; 9, Codimono; 10, C.S.; 11, Fibrante; 12, CINRO; 13, CINBOL.

The chemotype assessment was performed by molecular markers described by Pacifico et al. [15] in order to confirm the chemotypes of the 13 genotypes.

The amplification reaction, consisting of $10 \mathrm{ng}$ of DNA and the recombinant Taq DNA Polymerase (Invitrogen), was performed in a T-Gradient Thermocycler (Biometra) in a final volume of $25 \mu \mathrm{L}$.

$5 \mu \mathrm{L}$ of PCR products were checked after adding $1 \mu \mathrm{L}$ of $6 \times$ DNA loading dye (Thermo Fisher Scientific) by electrophoresis on $1 \%$ agarose gel (TAE buffer $1 \times$ ), stained with Gel Red (Biotum). 1Kb plus DNA Ladder (Invitrogen) was used as marker for amplicon size. 
DNA was visualized under UV light on a transilluminator and digitally photographed with AlphaImager HP (Alpha Innotech, Santa Clara, CA, USA).

\section{Appendix B}

Optimization of RT-qPCR primers for selective and quantitative amplification of target cannabinoid synthase genes.

Primer design and amplification reactions were optimized to distinguish the different cannabinoid synthases sequences, developing highly specific assays for gene detection and transcriptional analyses.

Gene-specific primer pairs were designed using the software Primer3. Since the different genes encoding for Cannabinoid Synthases share high similarity at the nucleotide level, primers were carefully designed in presence of SNPs differentiating genes, identified by sequences alignment, by keeping the SNPs at the $3^{\prime}$ end of the primers. The primer pairs designed and employed in this work are shown in Table 3 of the main text.

Primer specificity was verified in different ways as described elsewhere [44]. First, primer pairs were searched by BlastN against Cannabis sativa non-redundant database at NCBI. Second, the presence of a single PCR product after end-point PCR and the absence of non-target amplification were assessed. The PCR reactions were performed in a $10 \mu \mathrm{L}$ total volume, with $200 \mu \mathrm{M}$ dNTPs, 0.2 U Phusion DNA Polymerase (Thermo Scientific) and different concentrations of the specific primer pairs. As template, $0.5 \mathrm{pg}$ of plasmid DNA, $1.5 \mu \mathrm{L}$ of cDNA (diluted 1:10, corresponding to $2.5 \mathrm{ng}$ starting RNA) and $10 \mathrm{ng}$ DNA were used.

Different pJET1.2 plasmid vectors (CloneJET PCR Cloning Kit, Thermo Scientific) obtained as described in paragraph 2.5 of the main text, were used in this second step: one containing the specific gene targeted by the primer pair (positive control), and one or more plasmids containing other cannabinoids synthase gene sequences (negative controls).

PCR were performed following this condition: $98^{\circ} \mathrm{C}$ for $30 \mathrm{~s}, 30$ cycles of $10 \mathrm{~s}$ at $98^{\circ} \mathrm{C}, 15 \mathrm{~s}$ at variable temperatures, and $15 \mathrm{~s}$ at $72{ }^{\circ} \mathrm{C}$, followed by a final extension of $5 \mathrm{~min}$ at $72{ }^{\circ} \mathrm{C}$. In order to optimize the reaction to get a specific product, different primers concentrations and annealing temperature were tested until the best condition was found.

The reaction was considered optimized only when amplification band was present in the positive control and absent in the negative ones (Figures A2-A4).

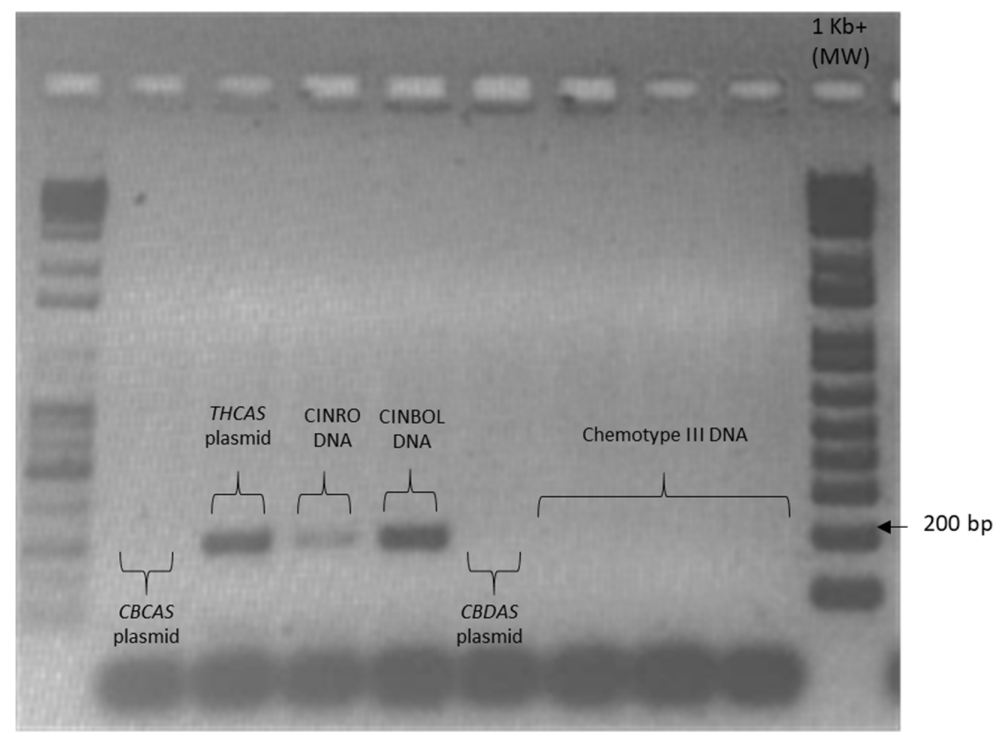

Figure A2. Specificity of primers for THCAS. The expected amplification band of $198 \mathrm{bp}$ was obtained from pJET1.2 containing the THCAS and DNA of CINRO and CINBOL. Amplification was not obtained from pJET1.2 plasmid containing the $C B C A S$, and the $C B D A S$, and neither using DNA from hemp varieties as template. 


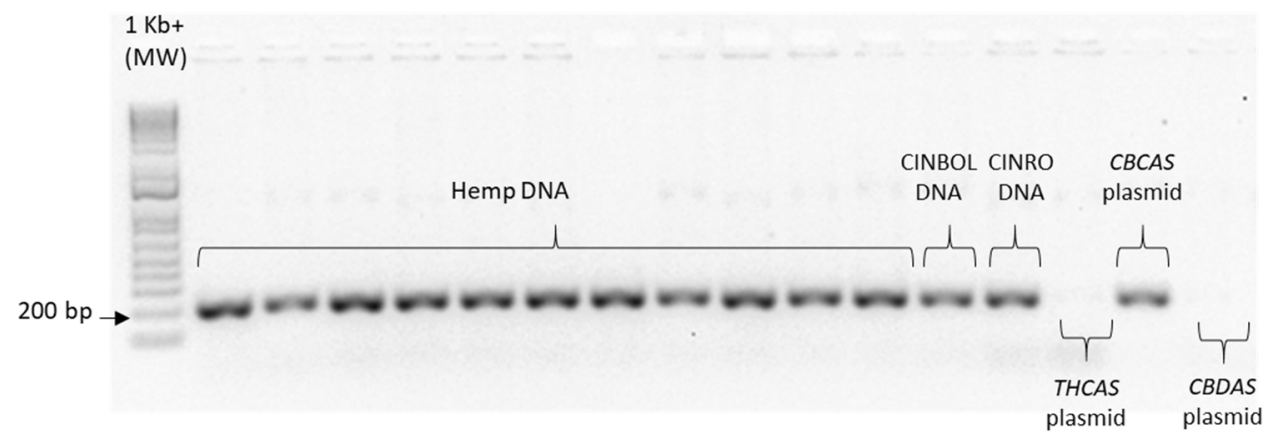

Figure A3. Specificity of primers for $C B C A S$ genes. The expected amplification band of $198 \mathrm{bp}$ was obtained from pJET1.2 containing the CBCAS and DNA of all tested genotypes. Amplification was not obtained from pJET1.2 plasmid containing the THCAS and the CBDAS, indicating a great specificity of this primer pairs.

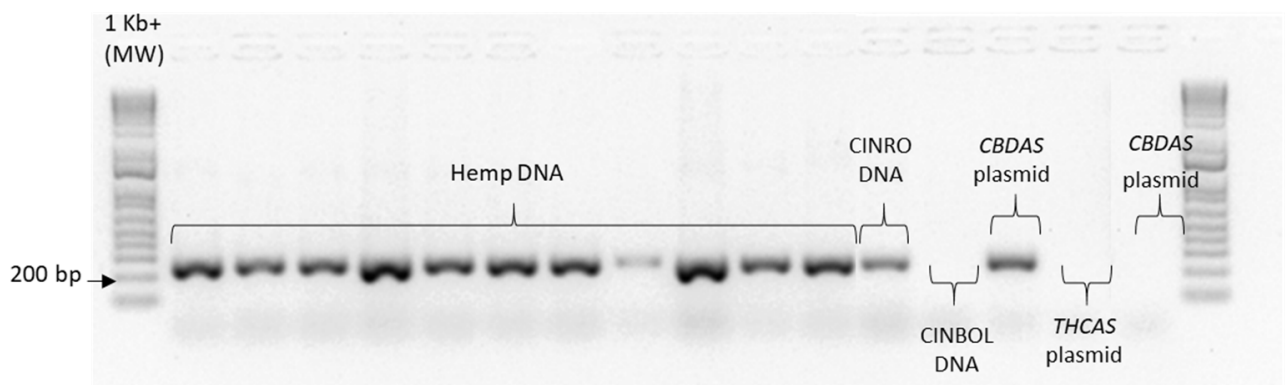

Figure A4. Specificity of primers for CBDAS. The expected amplification band of $241 \mathrm{bp}$ was obtained from pJET1.2 containing the CBDAS and DNA from hemp and from CINRO. Amplification was not obtained from pJET1.2 plasmid containing the THCAS and CBCAS. Primers work only on functional CBDAS sequences.

Primer pairs can be used as markers on DNA or to verify if the specific target gene is expressed using cDNA as template, as indicated in Figure A5.

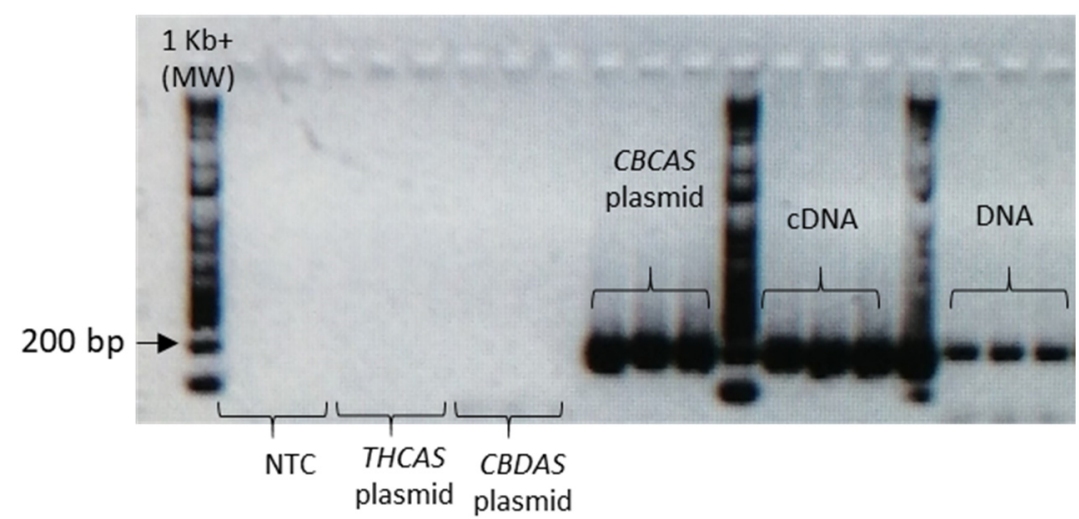

Figure A5. Agarose gel electrophoresis results of $C B C A S$ primers amplification on plasmids containing each cannabinoid synthases, genomic DNA and cDNA of a hemp plant in triplicate. The PCR assays contained also 2 negative controls, where water was added instead of template (lane 1 and 2).

Finally, primers specificity was tested by RT-qPCR with a RotorGene 6000 (Corbett). In this specificity assay, two parameters were changed to optimize the amplification conditions: the annealing temperature (from 58 to $62^{\circ} \mathrm{C}$ ) and the primers concentration (from $75 \mathrm{nM}$ to $1750 \mathrm{nM}$ ). Amplification reactions were conducted in a final volume of $10 \mu \mathrm{L}$ containing the Power Up ${ }^{\circledR}$ SYBR master mix (Thermo Fisher Scientific), primer pair (different 
concentrations) and RNase-free water. As template, cDNA, DNA and plasmid DNA were used as indicated above.

Different amplification conditions were used depending on the annealing temperature of the primers used: when this was below $60^{\circ} \mathrm{C}$, a two-step method was used, consisting of $15 \mathrm{~s}$ at variable temperature for annealing and $1 \mathrm{~min}$ at $72{ }^{\circ} \mathrm{C}$ for extension; when this was above $60^{\circ} \mathrm{C}$, a one-step method was used, consisting of $60 \mathrm{~s}$ at the desired temperature in which both annealing and extension are performed. Finally, to ensure the absence of not-specific PCR products and primer dimers, a heat dissociation protocol (from $60{ }^{\circ} \mathrm{C}$ to $99^{\circ} \mathrm{C}$ ) was performed and a dissociation curve for each sample was generated. Primers were considered as specific when they amplify a positive control and give no signal for negative controls (in both cases the controls are represented by plasmids with a known sequence). The production of a single melting curve peak in positive controls and samples suggested that the amplicon was specific (Figures A6-A8).

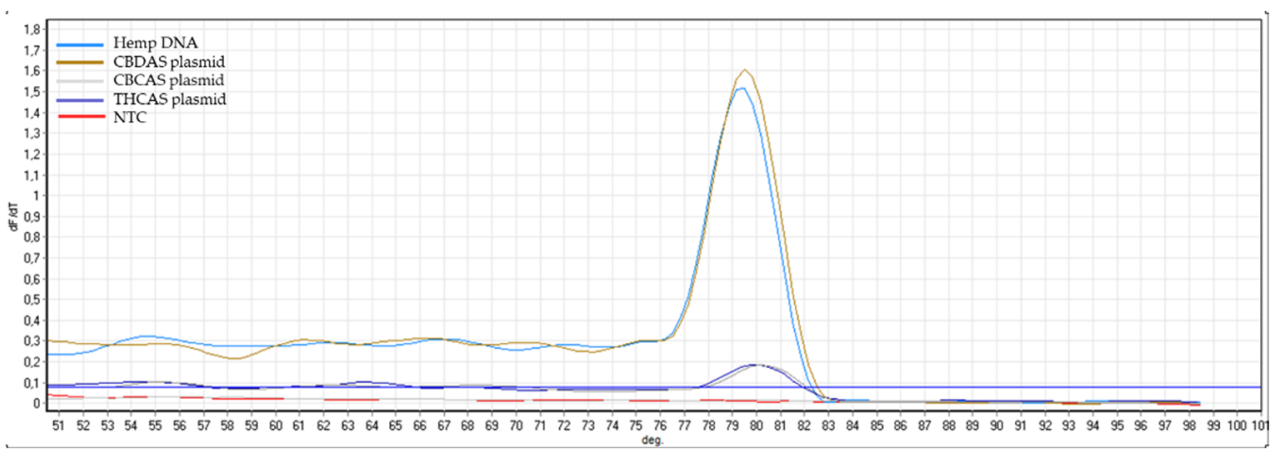

Figure A6. Melting curve plots, which display data collected during a melting curve stage with primers CBDAS_F and CBDAS_R. Peaks in the melting curve may indicate the melting temperature (Tm) of a target or identify nonspecific PCR amplification. No peaks were seen for the no template control $\left(\mathrm{H}_{2} \mathrm{O}\right)$ and for negative controls (THCAS plasmid and CBCAS plasmid).

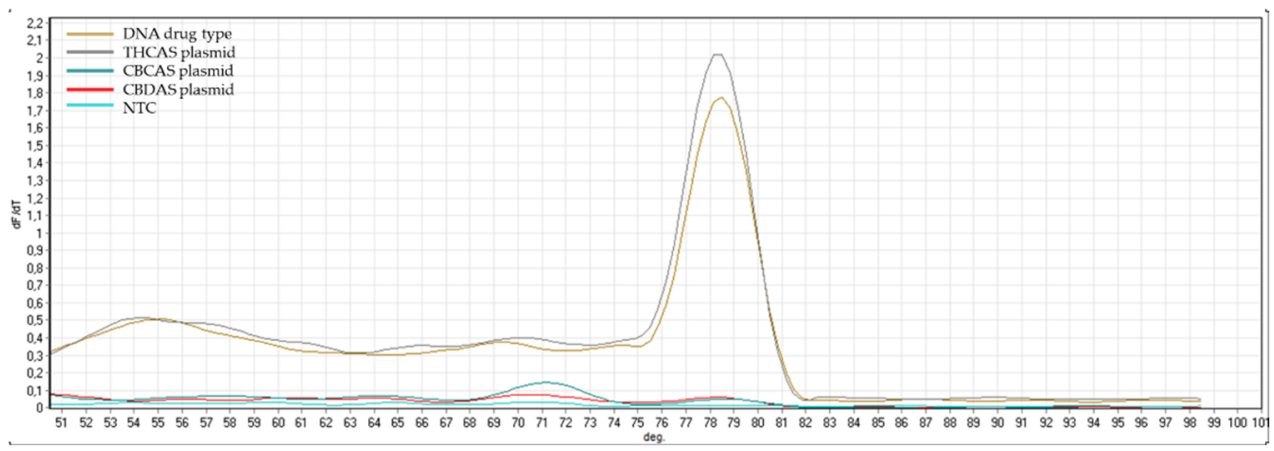

Figure A7. Melting curve plots, which display data collected during a melting curve stage with primers THCAS_F and THCAS_R. Peaks in the melting curve may indicate the melting temperature $(\mathrm{Tm})$ of a target or identify nonspecific PCR amplification. No peaks were observed for the no template control $\left(\mathrm{H}_{2} \mathrm{O}\right)$ and for negative controls (CBDAS plasmid and $C B C A S$ plasmid). 


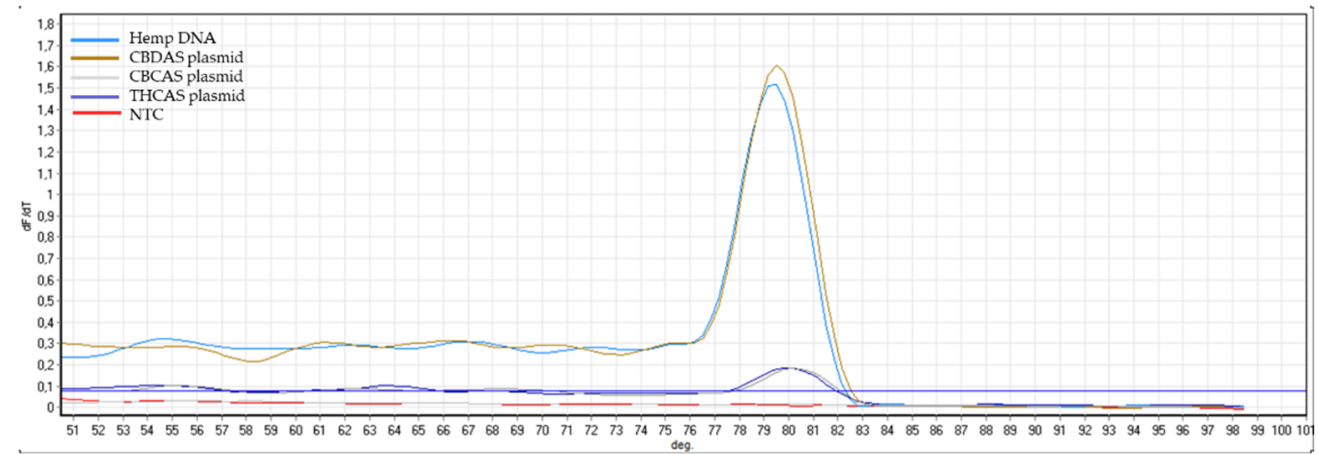

Figure A8. Melting curve plots, which display data collected during a melting curve stage with primers CBCAS_F and CBCAS_R. Peaks in the melting curve may indicate the melting temperature (Tm) of a target or identify nonspecific PCR amplification. No peaks were seen for the no template control $\left(\mathrm{H}_{2} \mathrm{O}\right)$ and for negative controls (THCAS plasmid, CBDAS plasmid).

\section{References}

1. Gülck, T.; Møller, B.L. Phytocannabinoids: Origins and Biosynthesis. Trends Plant Sci. 2020, 25, 985-1004. [CrossRef]

2. Mandolino, G.; Carboni, A. Potential of Marker-Assisted Selection in Hemp Genetic Improvement. Euphytica 2004, 140, 107-120. [CrossRef]

3. Sirikantaramas, S.; Morimoto, S.; Shoyama, Y.; Ishikawa, Y.; Wada, Y.; Shoyama, Y.; Taura, F. The Gene Controlling Marijuana Psychoactivity. Molecular Cloning and Heterologous Expression of $\Delta 1$-Tetrahydrocannabinolic Acid Synthase from Cannabis sativa L. J. Biol. Chem. 2004, 279, 39767-39774. [CrossRef]

4. Taura, F.; Morimoto, S.; Shoyama, Y. Purification and Characterization of Cannabidiolic-Acid Synthase from Cannabis sativa L. Biochemical Analysis of a Novel Enzyme That Catalyzes the Oxidocyclization of Cannabigerolic Acid to Cannabidiolic Acid. J. Biol. Chem. 1996, 271, 17411-17416. [CrossRef]

5. Shoyama, Y.; Tamada, T.; Kurihara, K.; Takeuchi, A.; Taura, F.; Arai, S.; Blaber, M.; Shoyama, Y.; Morimoto, S.; Kuroki, R. Structure and Function of $\Delta 1$-Tetrahydrocannabinolic Acid (THCA) Synthase, the Enzyme Controlling the Psychoactivity of Cannabis Sativa. J. Mol. Biol. 2012, 423, 96-105. [CrossRef]

6. $\quad$ Laverty, K.U.; Stout, J.M.; Sullivan, M.J.; Shah, H.; Gill, N.; Holbrook, L.; Deikus, G.; Sebra, R.; Hughes, T.R.; Page, J.E.; et al. A Physical and Genetic Map of Cannabis Sativa Identifies Extensive Rearrangements at the THC/CBD Acid Synthase Loci. Genome Res. 2019, 29, 146-156. [CrossRef]

7. van Velzen, R.; Schranz, M.E. Origin and Evolution of the Cannabinoid Oxidocyclase Gene Family. BioRxiv 2020. [CrossRef]

8. Kojoma, M.; Seki, H.; Yoshida, S.; Muranaka, T. DNA Polymorphisms in the Tetrahydrocannabinolic Acid (THCA) Synthase Gene in "Drug-Type" and "Fiber-Type" Cannabis sativa L. Forensic Sci. Int. 2006, 159, 132-140. [CrossRef] [PubMed]

9. Weiblen, G.D.; Wenger, J.P.; Craft, K.J.; ElSohly, M.A.; Mehmedic, Z.; Treiber, E.L.; Marks, M.D. Gene Duplication and Divergence Affecting Drug Content in Cannabis Sativa. New Phytol. 2015, 208, 1241-1250. [CrossRef]

10. Cascini, F.; Farcomeni, A.; Migliorini, D.; Baldassarri, L.; Boschi, I.; Martello, S.; Amaducci, S.; Lucini, L.; Bernardi, J. Highly Predictive Genetic Markers Distinguish Drug-Type from Fiber-Type Cannabis sativa L. Plants 2019, 8, 496. [CrossRef]

11. Hurgobin, B.; Tamiru-Oli, M.; Welling, M.T.; Doblin, M.S.; Bacic, A.; Whelan, J.; Lewsey, M.G. Recent Advances in Cannabis sativa Genomics Research. New Phytol. 2021, 230, 73-89. [CrossRef]

12. Morimoto, S.; Komatsu, K.; Taura, F.; Shoyama, Y. Purification and Characterization of Cannabichromenic Acid Synthase from Cannabis sativa. Phytochemistry 1998, 49, 1525-1529. [CrossRef]

13. de Meijer, E.P.M.; Hammond, K.M.; Micheler, M. The Inheritance of Chemical Phenotype in Cannabis sativa L. (III): Variation in Cannabichromene Proportion. Euphytica 2009, 165, 293-311. [CrossRef]

14. Borroto Fernandez, E.; Peterseil, V.; Hackl, G.; Menges, S.; de Meijer, E.; Staginnus, C. Distribution of Chemical Phenotypes (Chemotypes) in European Agricultural Hemp (Cannabis sativa L.) Cultivars. J. Forensic Sci. 2020, 65, 715-721. [CrossRef]

15. Pacifico, D.; Miselli, F.; Micheler, M.; Carboni, A.; Ranalli, P.; Mandolino, G. Genetics and Marker-Assisted Selection of the Chemotype in Cannabis sativa L. Mol. Breed. 2006, 17, 257-268. [CrossRef]

16. Staginnus, C.; Zörntlein, S.; de Meijer, E. A PCR Marker Linked to a THCA Synthase Polymorphism Is a Reliable Tool to Discriminate Potentially THC-Rich Plants of Cannabis sativa L. J. Forensic Sci. 2014, 59, 919-926. [CrossRef]

17. van Bakel, H.; Stout, J.M.; Cote, A.G.; Tallon, C.M.; Sharpe, A.G.; Hughes, T.R.; Page, J.E. The Draft Genome and Transcriptome of Cannabis sativa. Genome Biol. 2011, 12, R102. [CrossRef]

18. Gao, S.; Wang, B.; Xie, S.; Xu, X.; Zhang, J.; Pei, L.; Yu, Y.; Yang, W.; Zhang, Y. A High-Quality Reference Genome of Wild Cannabis sativa. Hortic. Res. 2020, 7, 73. [CrossRef] 
19. Grassa, C.J.; Weiblen, G.D.; Wenger, J.P.; Dabney, C.; Poplawski, S.G.; Motley, S.T.; Michael, T.P.; Schwartz, C.J. A New Cannabis Genome Assembly Associates Elevated Cannabidiol (CBD) with Hemp Introgressed into Marijuana. New Phytol. 2021, 230, 1665-1679. [CrossRef]

20. McKernan, K.J.; Helbert, Y.; Kane, L.T.; Ebling, H.; Zhang, L.; Liu, B.; Eaton, Z.; McLaughlin, S.; Kingan, S.; Baybayan, P.; et al. Sequence and Annotation of 42 Cannabis Genomes Reveals Extensive Copy Number Variation in Cannabinoid Synthesis and Pathogen Resistance Genes. BioRxiv 2020. [CrossRef]

21. Braich, S.; Baillie, R.C.; Jewell, L.S.; Spangenberg, G.C.; Cogan, N.O.I. Generation of a Comprehensive Transcriptome Atlas and Transcriptome Dynamics in Medicinal Cannabis. Sci. Rep. 2019, 9, 1-12. [CrossRef]

22. Bassolino, L.; Buti, M.; Fulvio, F.; Pennesi, A.; Mandolino, G.; Milc, J.; Francia, E.; Paris, R. In Silico Identification of MYB and BHLH Families Reveals Candidate Transcription Factors for Secondary Metabolic Pathways in Cannabis sativa L. Plants 2020, 9 , 1540. [CrossRef]

23. Liu, Y.; Zhu, P.; Cai, S.; Haughn, G.; Page, J.E. Three Novel Transcription Factors Involved in Cannabinoid Biosynthesis in Cannabis sativa L. Plant Mol. Biol. 2021, 106, 49-65. [CrossRef] [PubMed]

24. Mandolino, G.; Bagatta, M.; Carboni, A.; Ranalli, P.; de Meijer, E. Qualitative and Quantitative Aspects of the Inheritance of Chemical Phenotype in Cannabis. J. Ind. Hemp 2003, 8, 51-72. [CrossRef]

25. Onofri, C.; de Meijer, E.P.M.; Mandolino, G. Sequence Heterogeneity of Cannabidiolic- and Tetrahydrocannabinolic Acid-Synthase in Cannabis sativa L. and Its Relationship with Chemical Phenotype. Phytochemistry 2015, 116, 57-68. [CrossRef] [PubMed]

26. Vergara, D.; Huscher, E.L.; Keepers, K.G.; Givens, R.M.; Cizek, C.G.; Torres, A.; Gaudino, R.; Kane, N.C. Gene Copy Number Is Associated with Phytochemistry in Cannabis sativa. bioRxiv 2019. [CrossRef] [PubMed]

27. Zirpel, B.; Kayser, O.; Stehle, F. Elucidation of Structure-Function Relationship of THCA and CBDA Synthase from Cannabis sativa L. J. Biotechnol. 2018, 284, 17-26. [CrossRef]

28. Felsenstein, J. Confidence limits on phylogenies: An approach using the bootstrap. Evolution 1985, 39, 783-791. [CrossRef]

29. Tamura, K.; Nei, M.; Kumar, S. Prospects for inferring very large phylogenies by using the neighbor-joining method. Proc. Natl. Acad. Sci. USA 2004, 101, 11030-11035. [CrossRef]

30. Toth, J.A.; Stack, G.M.; Cala, A.R.; Carlson, C.H.; Wilk, R.L.; Crawford, J.L.; Viands, D.R.; Philippe, G.; Smart, C.D.; Rose, J.K.C.; et al. Development and Validation of Genetic Markers for Sex and Cannabinoid Chemotype in Cannabis sativa L. GCB Bioenergy 2020, 12, 213-222. [CrossRef]

31. Wenger, J.P.; Dabney, C.J.; ElSohly, M.A.; Chandra, S.; Radwan, M.M.; Majumdar, C.G.; Weiblen, G.D. Validating a Predictive Model of Cannabinoid Inheritance with Feral, Clinical, and Industrial Cannabis sativa. Am. J. Bot. 2020, 107, 1423-1432. [CrossRef]

32. Schluttenhofer, C.; Yuan, L. Challenges towards Revitalizing Hemp: A Multifaceted Crop. Trends Plant Sci. 2017, 22, 917-929. [CrossRef]

33. Booth, J.K.; Bohlmann, J. Terpenes in Cannabis sativa-From Plant Genome to Humans. Plant Sci. 2019, 284, 67-72. [CrossRef] [PubMed]

34. Zager, J.J.; Lange, I.; Srividya, N.; Smith, A.; Lange, B.M. Gene Networks Underlying Cannabinoid and Terpenoid Accumulation in Cannabis. Plant Physiol. 2019, 180, 1877-1897. [CrossRef] [PubMed]

35. Citti, C.; Ciccarella, G.; Braghiroli, D.; Parenti, C.; Vandelli, M.A.; Cannazza, G. Medicinal Cannabis: Principal Cannabinoids Concentration and Their Stability Evaluated by a High Performance Liquid Chromatography Coupled to Diode Array and Quadrupole Time of Flight Mass Spectrometry Method. J. Pharm. Biomed. Anal. 2016, 128, 201-209. [CrossRef] [PubMed]

36. Linciano, P.; Citti, C.; Luongo, L.; Belardo, C.; Maione, S.; Vandelli, M.A.; Forni, F.; Gigli, G.; Laganà, A.; Montone, C.M.; et al. Isolation of a High-Affinity Cannabinoid for the Human CB1 Receptor from a Medicinal Cannabis sativa Variety: $\Delta$ 9-Tetrahydrocannabutol, the Butyl Homologue of $\Delta$ 9-Tetrahydrocannabinol. J. Nat. Prod. 2020, 83, 88-98. [CrossRef]

37. Baratta, F.; Simiele, M.; Pignata, I.; Enri, L.R.; Torta, R.; de Luca, A.; Collino, M.; D’Avolio, A.; Brusa, P. Development of Standard Operating Protocols for the Optimization of Cannabis-Based Formulations for Medical Purposes. Front. Pharmacol. 2019, 10, 1-10. [CrossRef]

38. Citti, C.; Linciano, P.; Russo, F.; Luongo, L.; Iannotta, M.; Maione, S.; Laganà, A.; Capriotti, A.L.; Forni, F.; Vandelli, M.A.; et al. A Novel Phytocannabinoid Isolated from Cannabis sativa L. with an in Vivo Cannabimimetic Activity Higher than $\Delta$ 9-Tetrahydrocannabinol: $\Delta$ 9-Tetrahydrocannabiphorol. Sci. Rep. 2019, 9, 1-13. [CrossRef]

39. Linciano, P.; Citti, C.; Russo, F.; Tolomeo, F.; Lagan, A.; Capriotti, A.L.; Luongo, L.; Iannotta, M.; Belardo, C.; Maione, S.; et al. Identification of a New Cannabidiol n-Hexyl Homolog in a Medicinal Cannabis Variety with an Antinociceptive Activity in Mice: Cannabidihexol. Sci. Rep. 2020, 10, 22019. [CrossRef]

40. Koressaar, T.; Remm, M. Enhancements and Modifications of Primer Design Program Primer3. Bioinformatics 2007, 23, 1289-1291. [CrossRef]

41. Untergasser, A.; Cutcutache, I.; Koressaar, T.; Ye, J.; Faircloth, B.C.; Remm, M.; Rozen, S.G. Primer3—New capabilities and interfaces. Nucleic Acids Res. 2012, 40, e115. [CrossRef] [PubMed]

42. Kumar, S.; Stecher, G.; Li, M.; Knyaz, C.; Tamura, K. MEGA X: Molecular Evolutionary Genetics Analysis across Computing Platforms. Mol. Biol. Evol. 2018, 35, 1547-1549. [CrossRef] [PubMed]

43. Mangeot-Peter, L.; Legay, S.; Hausman, J.F.; Esposito, S.; Guerriero, G. Identification of Reference Genes for RT-QPCR Data Normalization in Cannabis sativa Stem Tissues. Int. J. Mol. Sci. 2016, 17, 1556. [CrossRef] [PubMed] 
44. Pagliarani, G.; Paris, R.; Arens, P.; Tartarini, S.; Ricci, G.; Smulders, M.M.J.; van de Weg, W.E. A QRT-PCR Assay for the Expression of All Mal d 1 Isoallergen Genes. BMC Plant Biol. 2013, 13, 51. [CrossRef]

45. Bustin, S.A.; Benes, V.; Garson, J.A.; Hellemans, J.; Huggett, J.; Kubista, M.; Mueller, R.; Nolan, T.; Pfaffl, M.W.; Shipley, G.L.; et al. The MIQE guidelines: Minimum information for publication of quantitative real-time PCR experiments. Clin. Chem. 2009, 55, 611-622. [CrossRef]

46. Combest, M.M.; Moroz, N.; Tanaka, K.; Rogan, C.J.; Anderson, J.C.; Thura, L.; Rakotondrafara, A.M.; Goyer, A. StPIP1, a PAMPinduced peptide in potato, elicits plant defenses and is associated with disease symptom severity in a compatible interaction with Potato virus Y. J. Exp. Bot. 2021, 72, 4472-4488. [CrossRef]

47. Xie, F.; Xiao, P.; Chen, D.; Xu, L.; Zhang, B. MiRDeepFinder: A MiRNA Analysis Tool for Deep Sequencing of Plant Small RNAs. Plant Mol. Biol. 2012, 80, 75-84. [CrossRef]

48. Larionov, A.; Krause, A.; Miller, W.R. A Standard Curve Based Method for Relative Real Time PCR Data Processing. BMC Bioinform. 2005, 6, 62. [CrossRef] 